\title{
La retorica biblica
}

\author{
A retórica bíblica
}

Roland Meynet

\section{Riassunto}

Questo articolo presenta la Retorica Biblica, come un Metodo di Analisi per testi biblici e non biblici, con applicazioni ed esempi lungo questo testo. Questo è un lavoro che è già stato presentato in altri momenti e in diverse lingue, con la finalità di poter espandere la conoscenza e l'applicazione della Retorica Biblica Semitica nell'ambiente accademico, presso le nostre istituzioni e centri di studio in Teologia Biblica. Lungi dall'essere un metodo nuovo o recente, 1'Analisi Retorica ha le sue basi nella retorica greca e in quella romana, oltre ad essere stata utilizzata nel corso dei secoli, come è ancora oggi. La novità è la riscoperta dell'Analisi Retorica come Analisi Retorica Semitica Biblica, ampiamente utilizzata nell'area geografica e culturale del Medio Oriente, territorio in cui fiorirono i testi biblici dell'AT e de NT, che fanno parte della vasta tradizione giudaico-cristiana. In questo senso, possiamo dire che è nei testi biblici che l'Analisi Retorica Semitica è stata recentemente riscoperta. Pertanto, vogliamo qui offrire le basi dell'Analisi Biblica Retorica Semitica, i suoi passi, figure e frutti, anche a partire della sua applicazione nei testi biblici, sia dell'AT che del NT, al fine di poter espandere la nostra conoscenza nel campo degli studi biblico.

Parole chiave: Analisi retorica. Retorica Biblica. Retorica Remitica. Bibbia. Parallelismo.

\section{Resumo}

Este artigo apresenta a Retórica Bíblica, enquanto Método de Análise 
para textos bíblicos e não bíblicos, com aplicação e exemplos ao longo deste texto. Este é um trabalho que já foi apresentado em outros momentos e em diferentes línguas, com a finalidade de se poder ampliar o conhecimento e aplicação da Retórica Bíblica Semítica no meio acadêmico, junto às nossas instituiões e centros de estudos em Teologia Bíblica. Longe de ser um método novo ou recente, a Análise Retórica tem seus fundamentos na retórica grega e e na retórica romana, além de ter sido sempre usada aos longo dos séculos, como ainda hoje. A novidade é a redescoberta da Análise Retórica enquanto Análise Retórica Biblica Semítica, usada amplamente na área gerográfica e cultural do Oriete Médio, território onde floreceram os textos bíblicos do AT e do NT, que fazem parte da ampla tradição judaico-cristã. Neste sentido, podemos dizer que é nos textos bíblicos que a Análise Retórica Semítica foi redescoberta recentemente. Por isso, queremos aqui, ofercer as bases da Análise Retórica Bíblica Semítica, seus passos, figuras e frutos, inclusive a partir de sua aplicação em textos bíblicos, tanto do AT como do NT, a fim de poder ampliar nossos conhecimentos na área dos estudos bíblicos.

Palavras-chave: Análise Retórica. Retórica Bíblica. Retórica Semítca. Bíblia. Paralelismo.

\section{Introduzione}

Non è la prima volta che presento la retorica biblica in modo sintetico nei limiti di un articolo. Il primo che ho pubblicato sull'argomento raccontava sostanzialmente la storia della sua scoperta. ${ }^{1}$ Quindi, su richiesta di una rivista portoghese, ne ho fatto una presentazione per il grande pubblico, ${ }^{2}$ che è stata poi tradotta in italiano, francese, arabo, inglese, giapponese, polacco e spagnolo. ${ }^{3}$ Dopo di che ci sono tornato più volte, in diverse forme. ${ }^{4}$

La prima cosa da precisare è che bisogna distinguere tra retorica biblica e retorica classica, greco-latina, insomma la nostra retorica occidentale.

\footnotetext{
${ }^{1}$ MEYNET, R., Histoire de l'analyse rhétorique en exégèse biblique, p. 291-320.

${ }^{2}$ MEYNET, R., A Análise retórica, p. 391-408.

${ }^{3}$ ONISZCZUK, J., L'analisi retorica biblica e semitica, p. 479-501.

${ }^{4}$ MEYNET, R., La rhétorique biblique et sémitique, p. 290-312; MEYNET, R., La rhétorique biblique et sémitique, p. 44-77; MEYNET, R., Rhétorique biblique, rhétorique de l'énigme, p. 147-180; MEYNET, R., Une nouvelle présentation de la rhétorique biblique et sémitique.
} 


\section{La retorica classica, occidentale}

Questa retorica si chiama classica perché è quella che abbiamo appreso da più di venti secoli e fino ad oggi nelle classi, cioè le aule scolastiche, dalla scuola elementare all'università. La nostra retorica nacque in Grecia nel IV secolo aC, con Aristotele. È nata dall'esperienza degli oratori che parlavano sull'agorà, il luogo pubblico in cui erano discusse le questioni politiche, e anche in tribunale dove uno doveva difendere la propria causa. In entrambi i casi, si trattava di "convincere" gli ascoltatori e di piacere per convincere meglio. ${ }^{5}$

Questo è esattamente ciò che continuiamo a fare oggi, non solo nelle scuole di Scienze politiche dove si formano i politici, ma anche nei corsi di formazione dei partiti e dei sindacati. Al liceo si impara a scrivere una dissertazione: i professori insegnano come comporre un'introduzione (catturare l'attenzione del lettore, annunciare la divisione o il piano della presentazione), quindi $l o$ svolgimento (spesso in tre fasi: tesi, antitesi, sintesi) infine la conclusione (che include la ricapitolazione, e deve aprire il discorso sui possibili sviluppi). Il discorso, come in matematica, è lineare: dalle premesse, il ragionamento progredisce con tutta una serie di argomenti o prove, fino alla conclusione in cui il lettore trova chiaramente espresso il punto in cui l'oratore voleva guidarlo, per guadagnare il suo consenso, l'adesione alle sue idee. Quindi, a grandi linee, questa è la retorica classica occidentale, quella che gradualmente ha imposto le sue regole in tutto il mondo.

\section{La retorica biblica, semitica}

Contrariamente a quanto pensano molti occidentali, la retorica classica non è l'unica al mondo! Un proverbio africano dice: "Chi non ha mai lasciato il suo villaggio, crede che solo sua madre sappia preparare la minestra". Il "villaggio" in questione in questo caso può chiamarsi Parigi, New York o Berlino... Vi è anche, tra le altre, una retorica biblica, e più ampiamente semitica, vale a dire usata nell'intera area geografica e culturale del Vicino Oriente. Lasciamo da parte i testi babilonesi, ugaritici o antichi egiziani e concentriamoci sulla Bibbia. Semplicemente perché è nei testi biblici che è stata scoperta questa retorica specifica.

${ }^{5}$ MORTARA GARAVELLI, B., Manuale di retorica. 
Ho detto "scoperta"! Sì, perché quella retorica non faceva parlare di se stessa; ed è per questo che non se ne parlava, o molto poco fino ad una data relativamente recente. E anche perché si voleva trovare nella letteratura biblica le stesse regole che nella retorica classica greco-latina. Ad esempio, persone rispettabili come Flavio Giuseppe e Origene pensavano di trovare pentametri o esametri nella poesia ebraica (come se dicessero ottonari o endecasillabi, quei versi italiani che hanno otto o undici sillabe!). ${ }^{6}$ Ma il verso ebraico non obbedisce alle leggi della poesia greca o latina, né a quelle della poesia classica italiana. Dobbiamo uscire risolutamente dal piccolo villaggio ... Diciamo "scoperta", perché, a differenza del mondo greco-romano, nessun trattato di retorica è stato composto nel mondo biblico e semitico. Dunque, questa retorica e le sue leggi hanno dovuto essere scoperte dai ricercatori.?

\section{La scoperta fondamentale: il parallelismus membrorum}

La scoperta della struttura del verso ebraico è attribuita a un professore di poesia, Robert Lowth, che insegnò all'Università di Oxford nel diciottesimo secolo. Nel 1753 pubblicò le sue Lezioni sulla sacra poesia degli Ebrei. ${ }^{8}$ Ciò che i posteri hanno universalmente ritenuto è la sua diciannovesima lezione su ciò che egli chiama "il parallelismo dei membri". Ecco il primo esempio che dà:

\begin{tabular}{|c|c|c|c|}
\hline $\begin{array}{l}{ }^{1} b^{\mathrm{e}} \mathrm{sse}_{\mathrm{e}}{ }^{\prime} \mathrm{t} \\
\text { bêt }\end{array}$ & 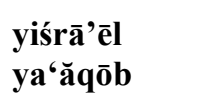 & $\begin{array}{l}\text { mimmișrāyim, } \\
\text { mē'am }\end{array}$ & 1ō‘èz \\
\hline $\begin{array}{l}\text { Quando uscì } \\
\text { casa }\end{array}$ & $\begin{array}{l}\text { Israele } \\
\text { di Giacobbe }\end{array}$ & $\begin{array}{l}\text { dall'Egitto, } \\
\text { da un popolo }\end{array}$ & niero $($ Sal 114,1) \\
\hline
\end{tabular}

Come possiamo vedere, questo primo tipo di "verso" ebraico è composto da due "membri" che, con parole diverse, dicono praticamente la stessa cosa: "la casa di Giacobbe" è un altro modo di dire "Israele", perché "Giacobbe", figlio di Isacco, ricevette dall'angelo con cui combatté tutta la notte il nome

\footnotetext{
${ }^{6}$ KUGEL, J. K., The Idea of Biblical Poetry, p. 149-156.

${ }^{7}$ MEYNET, R., Première partie, p. 23-173; ripreso e abbreviato nel mio MEYNET, R., Historique, p. 31-110; MEYNET, R., Histoire de l'analyse rhétorique en exégèse biblique, p. 291-320; e soprattutto, MEYNET, R., La rhétorique biblique et sémitique, p. 290-312.

${ }^{8}$ LOWTH, R., De sacra poesi Hebraeorum.
} 
di "Israele" (Gen 32,23-33); inoltre, "la casa di Giacobbe" significa la sua famiglia, il suo "casato", vale a dire i suoi dodici figli, quelli che hanno dato il loro nome alle dodici tribù di Israele.

Lo stesso vale per i seguenti versi, come possiamo vedere in questa riscrittura:

\begin{tabular}{|c|c|c|}
\hline $\begin{array}{l}{ }^{3} \text { hayyām } \\
\text { hayyardēn }\end{array}$ & $\begin{array}{l}\text { rā'â } \\
\text { yissōb }\end{array}$ & 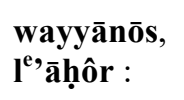 \\
\hline $\begin{array}{l}{ }^{4} \text { hehārîm } \\
g^{e} b \bar{a} \text { 'ôt }\end{array}$ & $\begin{array}{l}\text { rāqdû } \\
\text { kibnê- }\end{array}$ & $\begin{array}{l}\boldsymbol{k}^{e}, \hat{e} l \hat{i} m, \\
\text { șō'n : }\end{array}$ \\
\hline $\begin{array}{l}{ }^{5} \text { mah- }-l^{\mathrm{e}} \mathrm{ka} \\
\text { hayyardēn }\end{array}$ & $\begin{array}{l}\text { hayyām } \\
\text { tissōb }\end{array}$ & $\begin{array}{l}\text { kî tānûs, } \\
\text { le' }^{\mathrm{e}} \bar{a} h \underline{o ̂} \mathbf{r}:\end{array}$ \\
\hline $\begin{array}{l}{ }^{6} \text { hehārî̀m } \\
g^{e} b \bar{a} \text { 'ôt }\end{array}$ & $\begin{array}{l}\operatorname{tirq}^{\mathrm{e}} \mathrm{du} \\
\boldsymbol{k i b n} \hat{\boldsymbol{e}}-\end{array}$ & $\begin{array}{l}\boldsymbol{k}^{e}, \hat{e} l \hat{\imath} m, \\
\text { șō'n : }\end{array}$ \\
\hline $\begin{array}{l}{ }^{3} \text { Il mare } \\
\text { Il Giordano }\end{array}$ & $\begin{array}{l}\text { vide } \\
\text { tornò }\end{array}$ & $\begin{array}{l}\text { e fuggì, } \\
\text { indietro. }\end{array}$ \\
\hline $\begin{array}{l}{ }^{4} \text { Le montagne } \\
\text { le colline }\end{array}$ & $\begin{array}{l}\text { saltarono } \\
\text { come figli }\end{array}$ & $\begin{array}{l}\text { come arieti, } \\
\text { del gregge. }\end{array}$ \\
\hline $\begin{array}{l}{ }^{5} \text { Che hai tu, } \\
\text { Giordano, }\end{array}$ & $\begin{array}{l}\text { mare, } \\
\text { a tornare }\end{array}$ & $\begin{array}{l}\text { a fuggire, } \\
\text { indietro? }\end{array}$ \\
\hline $\begin{array}{l}{ }^{6} \text { Montagne, } \\
\text { colline, }\end{array}$ & $\begin{array}{l}\text { a saltare } \\
\text { come figli }\end{array}$ & $\begin{array}{l}\text { come arieti, } \\
\text { del gregge? }\end{array}$ \\
\hline
\end{tabular}

Si percepisce il ritmo originale dell'ebraico: ogni membro comprende tre termini o tre "accenti". I due membri di ciascuno di questi versi - che oggi sono chiamati "segmenti" - sono complementari: "il mare" è il Mar Rosso che Israele ha attraversato all'inizio dell'esodo, lasciando l'Egitto, il paese della schiavitù; "Il Giordano" è il fiume che ha attraversato alla fine dell'esodo per entrare nella terra promessa, la terra di Canaan. "Le montagne" sono grandi come gli "arieti" e "le colline" sono piccole come gli agnelli che il testo chiama letteralmente "i figli degli ovini". "Il mare" è grande, il fiume Giordano è piccolo.

Tutti questi segmenti, Lowth li chiama "sinonimici", ma esiste una seconda categoria di parallelismo, "il parallelismo antitetico", in cui i due membri si oppongono: 


$\begin{array}{llll}\begin{array}{l}\text { ne'ĕmānîm } \\ \text { wéna'tārôt }\end{array} & \begin{array}{l}\text { piș'ê } \\ \text { n'šî́qôt }\end{array} & \begin{array}{l}\text { 'ôhēb } \\ \text { śôné' }\end{array} \\ \begin{array}{l}\text { Sono fedeli } \\ \text { ma ingannevoli }\end{array} & \begin{array}{l}\text { le ferite } \\ \text { i baci }\end{array} & \begin{array}{l}\text { dell'amico } \\ \text { del nemico }\end{array} & (\operatorname{Pr} 27,6)\end{array}$

E infine una terza categoria, il "parallelismo sintetico", ove la costruzione dei due membri è simile, senza essere né sinonimici né antitetici, ma complementari:

$\begin{array}{lll}\begin{array}{l}\text { 'ahat } \\ \text { stayim- }\end{array} & \begin{array}{l}\text { dibber } \\ \text { zû }\end{array} & \begin{array}{l}\text { 'ělōhîm } \\ \text { šāmā'tî̀ }\end{array} \\ \begin{array}{l}\text { Una volta } \\ \text { due volte }\end{array} & \begin{array}{l}\text { ha parlato } \\ \text { questo }\end{array} & \begin{array}{l}\text { Dio, } \\ \text { ho sentito (Sal 62,12). }\end{array}\end{array}$

Questa tipologia che enumera tre tipi di parallelismo - sinonimico, antitetico e sintetico o costruttivo - è quella che viene menzionata nei dizionari. Ma c'è un'altra tipologia, meno apparente, che distingue il "bicolon", il "tricolon" e anche il "monocolon", secondo il numero di cola (il colon è il nome greco di ciò che il latino chiama il membrum) che contiene l'unità superiore al membro e alla quale Lowth non assegna un nome. Nella terminologia dell'"analisi retorica biblica", questa unità è chiamata il "segmento".

Lowth ha quindi notato - nelle pagine dedicate al parallelismo sinonimico, poi all'interno delle pagine dedicate al parallelismo sintetico che non ci sono solo segmenti a due membri (i segmenti bimembri), ma anche segmenti a tre membri (i “trimembri”):

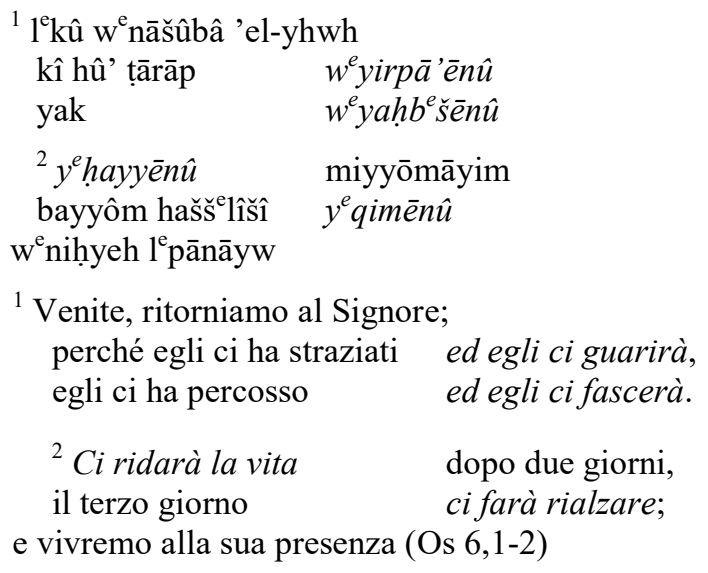


Lowth ha anche rilevato l'esistenza del segmento "unimembro", che nel seguente esempio si trova tra due segmenti bimembri (egli chiama l'insieme "pentacolon"):

\begin{tabular}{|c|c|c|}
\hline $\begin{array}{l}\text { tēre' } \\
\mathrm{w}^{\mathrm{e}} \boldsymbol{c} \boldsymbol{a} \boldsymbol{z} \boldsymbol{z} \hat{\boldsymbol{a}}\end{array}$ & $\begin{array}{l}\text { 'ašq }{ }^{e} \mathbf{l o ̂ n} \\
\text { wetāḥ̂îl }\end{array}$ &  \\
\hline $\mathrm{w}^{\mathrm{e}}$ 'eqrôn & kî-hōbîš & mebbāṭāh \\
\hline $\begin{array}{l}\mathrm{w}^{\mathrm{e}}, \bar{a} b a d \\
\text { we}^{\mathrm{e}} \text { 'ašqq }{ }^{\mathrm{e}} \mathbf{l} \mathbf{l} \mathbf{n}\end{array}$ & $\begin{array}{l}\text { melek } \\
\text { lō' tēšēb }\end{array}$ & mē'azzâ \\
\hline $\begin{array}{l}\text { Vedrà } \\
\text { e } \boldsymbol{G a z a},\end{array}$ & $\begin{array}{l}\text { Ascalon } \\
\text { e soffrirà }\end{array}$ & $\begin{array}{l}\text { e temerà, } \\
\text { molto. }\end{array}$ \\
\hline
\end{tabular}

Ed Ekron, perché svanirà la sua fiducia.

E perirà il re di Gaza

e Ascalon non sarà più abitata $\quad(\mathrm{Zc} 9,5)$.

Queste sono le basi di ciò che si sarebbe sviluppato in seguito e ora è chiamato "retorica biblica".

Prima di andare oltre, bisogna dire che la scoperta di Lowth risale molto più in là della metà del diciottesimo secolo: infatti, il professore di Oxford rimanda, citandolo ampiamente, a un rabbino italiano del sedicesimo secolo, Rabbi Azarias dei Rossi, che aveva evidenziato il sistema della poesia ebraica. E si potrebbe risalire ancora molto di più. La consapevolezza del parallelismo dei membri è certamente molto antica.

Una volta resi consapevoli dell'esistenza del parallelismo dei membri, possiamo costatare che si trova ovunque, ad esempio nei Salmi. Basta aprire il libro e leggere, ad esempio, il Sal 44, quello che inizia con il seguente segmento:

$\begin{array}{lll}\begin{array}{l}\text { 2,ělōhîm } \\ \text { 'ăbôtênû }\end{array} & \begin{array}{l}\mathrm{b}^{\mathrm{e}} \text { 'oznênû } \\ \text { sipp } \mathrm{e} \text { rû - }\end{array} & \begin{array}{l}\text { šāma'nû } \\ \text { lānû }\end{array} \\ { }^{2} \text { O Dio, } & \begin{array}{l}\text { con i nostri orecchi } \\ \text { hanno raccontato }\end{array} & \begin{array}{l}\text { abbiamo sentito, } \\ \text { i nostri padri... }\end{array}\end{array}$

e che prosegue fino alla fine con dei segmenti bimembri, sinonimici, antitetici e sintetici. 
Ma il parallelismo dei membri si trova anche nei profeti e persino nei testi narrativi sia dell'Antico Testamento sia del Nuovo Testamento.

\section{Lo sviluppo: composizioni parallele e concentriche}

\subsection{Le composizioni parallele}

Lowth aveva visto che due segmenti bimembri potevano formare un insieme (che egli chiama "tetracolon") di composizione parallela!

\begin{tabular}{|c|c|c|}
\hline $\begin{array}{l}{ }^{3} \text { miššāamayim } \\
\text { ră’âa }\end{array}$ & $\begin{array}{l}\text { HIBBîṬ } \\
\text { 'et kol-b' nê }\end{array}$ & $\begin{array}{l}\text { Yhwh } \\
\text { hā'ādām }\end{array}$ \\
\hline $\begin{array}{l}{ }^{14} \operatorname{mimm}^{e} k \hat{o} n \\
\text { 'el kol-yōšbê }\end{array}$ & $\begin{array}{l}\text { šibetô } \\
\text { hā'āreș }\end{array}$ & HIŠGÎAH \\
\hline $\begin{array}{l}{ }^{13} \text { Dai cieli } \\
\text { vede }\end{array}$ & $\begin{array}{l}\text { GUARDA } \\
\text { tutti i figli }\end{array}$ & $\begin{array}{l}\text { il Signore; } \\
\text { di Adamo; }\end{array}$ \\
\hline $\begin{array}{l}{ }^{14} \text { Dal luogo } \\
\text { tutti gli abitanti }\end{array}$ & $\begin{array}{l}\text { della sua dimora } \\
\text { della terra }\end{array}$ & CONTEMPLA \\
\hline
\end{tabular}

(Sal 33,13-14).

Per meglio far vedere le corrispondenze, abbiamo aggiunto gli allineamenti verticali dei termini e le differenze di caratteri. Ecco un altro esempio, sempre fornito da Lowth:

\begin{tabular}{|c|c|c|}
\hline $\begin{array}{c}{ }^{5} \text { kî bō'ălayik } \\
\text { Yhwh }\end{array}$ & $\begin{array}{l}\text { ‘ŌŚAYIK } \\
s^{e} b \bar{a} \text { 'ot }\end{array}$ & še mô \\
\hline $\begin{array}{l}\text { we gēóălēk } \\
\text { 'élohhê }\end{array}$ & $\begin{array}{l}\mathrm{Q}^{\mathrm{E}} \mathrm{DÔS} \\
\text { kol-hā'areses }\end{array}$ & $\begin{array}{l}\text { YIŚRĀ' }{ }^{\prime} \bar{L} \\
\text { yiqqāare' }\end{array}$ \\
\hline $\begin{array}{l}{ }^{5} \text { Perché tuo sposo } \\
\text { Signore }\end{array}$ & $\begin{array}{l}\text { TUO CREATORE, } \\
\text { degli eserciti }\end{array}$ & il suo nome. \\
\hline $\begin{array}{l}\text { E il tuo redentore } \\
\quad \text { Dio }\end{array}$ & $\begin{array}{l}\text { IL SANTO } \\
\text { di tutta la terra }\end{array}$ & $\begin{array}{l}\text { D'ISRAELE, } \\
\text { sarà chiamato }\end{array}$ \\
\hline
\end{tabular}

Negli ultimi due casi, i due segmenti formano un "brano" (nella terminologia della retorica biblica).

Lowth avrebbe potuto fare un altro passo in avanti, notando la composizione parallela dei quattro segmenti bimembri del suo primo esempio (Sal 114,3-6): 


\begin{tabular}{|c|c|c|c|}
\hline$={ }^{3}=$ & $\begin{array}{l}\text { HAYYĀM } \\
\text { hayyardēn }\end{array}$ & $\begin{array}{l}\text { rā'â } \\
\text { yissōb }\end{array}$ & 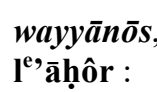 \\
\hline$:{ }^{4}$ & $\begin{array}{l}H E H \bar{A} R \hat{I} M \\
g^{e} b \bar{a} ' \hat{o} t\end{array}$ & $\begin{array}{l}\text { rāqdû } \\
\text { kibnê- }\end{array}$ & $\begin{array}{l}\text { k'êlêm, } \\
\text { șō'n : }\end{array}$ \\
\hline$=^{5}=$ & $\begin{array}{l}\text { mah-1 }{ }^{\mathrm{e}} \mathrm{k} \overline{\mathrm{a}} \\
\text { hayyardēn }\end{array}$ & $\begin{array}{l}\text { HAYYĀM } \\
\text { tissōb }\end{array}$ & $\begin{array}{l}\text { kî tānîs, } \\
\text { le, }^{\mathrm{e}} \mathbf{a} h \hat{o} \mathbf{r}:\end{array}$ \\
\hline$:{ }^{6}$ & $\begin{array}{l}H E H \bar{A} R \hat{I} M \\
g^{e} b \bar{a}{ }^{\prime} \hat{o} t\end{array}$ & $\begin{array}{l}\operatorname{tirq}^{\mathrm{e}} \text { dû } \\
\text { kibnê- }\end{array}$ & $\begin{array}{l}\text { k'êlîm, } \\
\text { șō'n : }\end{array}$ \\
\hline
\end{tabular}

\begin{tabular}{|c|c|c|c|}
\hline $\begin{array}{r}={ }^{3} \text { Il } \\
=\text { il }\end{array}$ & $\begin{array}{l}\text { MARE } \\
\text { Giordano }\end{array}$ & $\begin{array}{l}\text { vide } \\
\text { tornò }\end{array}$ & $\begin{array}{l}\text { e fuggì, } \\
\text { indietro, }\end{array}$ \\
\hline $\begin{array}{l}:{ }^{4} \text { le } \\
\quad: \text { le }\end{array}$ & $\begin{array}{l}\text { MONTAGNE } \\
\text { colline }\end{array}$ & $\begin{array}{l}\text { SALTARONO } \\
\text { come figli }\end{array}$ & $\begin{array}{l}\text { come arieti, } \\
\text { del gregge. }\end{array}$ \\
\hline$={ }^{5}=$ & $\begin{array}{l}\text { Che hai tu, } \\
\text { Giordano, }\end{array}$ & $\begin{array}{l}\text { MARE, } \\
\text { a tornare }\end{array}$ & $\begin{array}{l}\text { a fuggire, } \\
\text { indietro, }\end{array}$ \\
\hline$:^{6}:$ & $\begin{array}{l}\text { MONTAGNE, } \\
\text { colline, }\end{array}$ & $\begin{array}{l}\text { a SALTARE } \\
\text { come figli }\end{array}$ & $\begin{array}{l}\text { come arieti, } \\
\text { del gregge? }\end{array}$ \\
\hline
\end{tabular}

Sono infatti due brani, ciascuno formato da due segmenti bimembri, che costituiscono una "parte". Quindi, con questo esempio, abbiamo tre livelli, quello dei "segmenti" (qui solo bimembri), quello dei "brani" (qui ognuno formato da due segmenti), e infine quello della "parte" (che qui comprende due brani).

\subsection{Le composizioni concentriche}

Lowth ha anche notato la composizione concentrica. Riprendiamo lo stesso esempio:

$$
\begin{aligned}
& +{ }^{5} \text { Vedrà Ascalon e temerà, } \\
& \text { :: e Gaza, e soffrirà molto. } \\
& =\text { Ed EKRON, perché svanirà la sua fiducia. } \\
& \text { :: E perirà il re di Gaza } \\
& + \text { e Ascalon non sarà più abitata } \quad(Z c 9,5) \text {. }
\end{aligned}
$$


I nomi delle città filistee si corrispondono in modo concentrico: agli estremi "Ascalon", "Gaza" in seconda e penultima posizione, e infine "Ekron" sola al centro.

I segni tipografici posti all'inizio di ogni membro aiutano a visualizzare meglio la composizione: "più" all'inizio e alla fine, "doppio due punti" dopo e "uguale" al centro.

Saranno due ricercatori degli anni 1820-25 che, sulle fondamenta poste da Lowth, costruiranno il primo edificio della retorica biblica. Il primo era irlandese, John Jebb, ${ }^{9}$ e il secondo inglese, Thomas Boys. ${ }^{10}$ Nella tradizione dell'empirismo anglosassone, hanno notato che, a tutti i livelli, molti testi biblici sono composti in parallelo o in modo concentrico. ${ }^{11}$

Ecco un primo esempio, molto semplice, di composizione parallela: ${ }^{12}$

\begin{tabular}{|c|c|}
\hline $\begin{array}{l}\text { Ai } \tau \epsilon \hat{\imath} \tau \epsilon \\
\zeta \eta \tau \hat{\imath} \tau \epsilon \\
\kappa \rho o v \in \epsilon \epsilon \epsilon\end{array}$ & 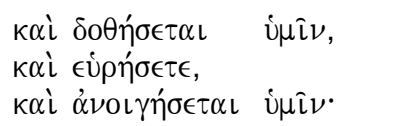 \\
\hline 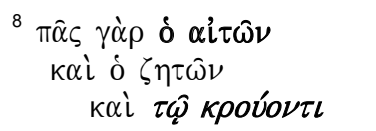 & 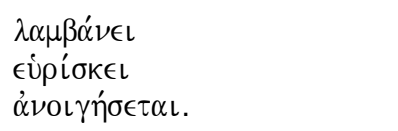 \\
\hline $\begin{array}{l}{ }^{7} \text { Chiedete } \\
\text { cercate } \\
\text { bussate }\end{array}$ & $\begin{array}{ll}\text { e sarà dato } & \text { a voi, } \\
\text { e troverete, } & \\
\text { e apriranno } & \text { a voi; }\end{array}$ \\
\hline $\begin{array}{c}{ }^{8} \text { Perché chiunque chiede } \\
\text { chi cerca } \\
\text { e } \boldsymbol{a} \text { chi bussa, }\end{array}$ & $\begin{array}{l}\text { riceve, } \\
\text { trova, } \\
\text { sarà aperto }\end{array}$ \\
\hline
\end{tabular}

\footnotetext{
${ }^{9}$ JEBB, J., Sacred Literature.

${ }^{10}$ BOYS, T., Tactica Sacra; BOYS, T., A Key to the Book of the Psalms.

${ }^{11}$ LOWTH, R., De Sacra Poesi Hebraeorum; JEBB, J., Sacred Literature; BOYS, T., Tactica Sacra; FORBES, J., The Symmetrical of Scripture.

12 JEBB, J., Sacred Literature, p. 156.
} 
Eccone un altro, molto più sviluppato: ${ }^{13}$

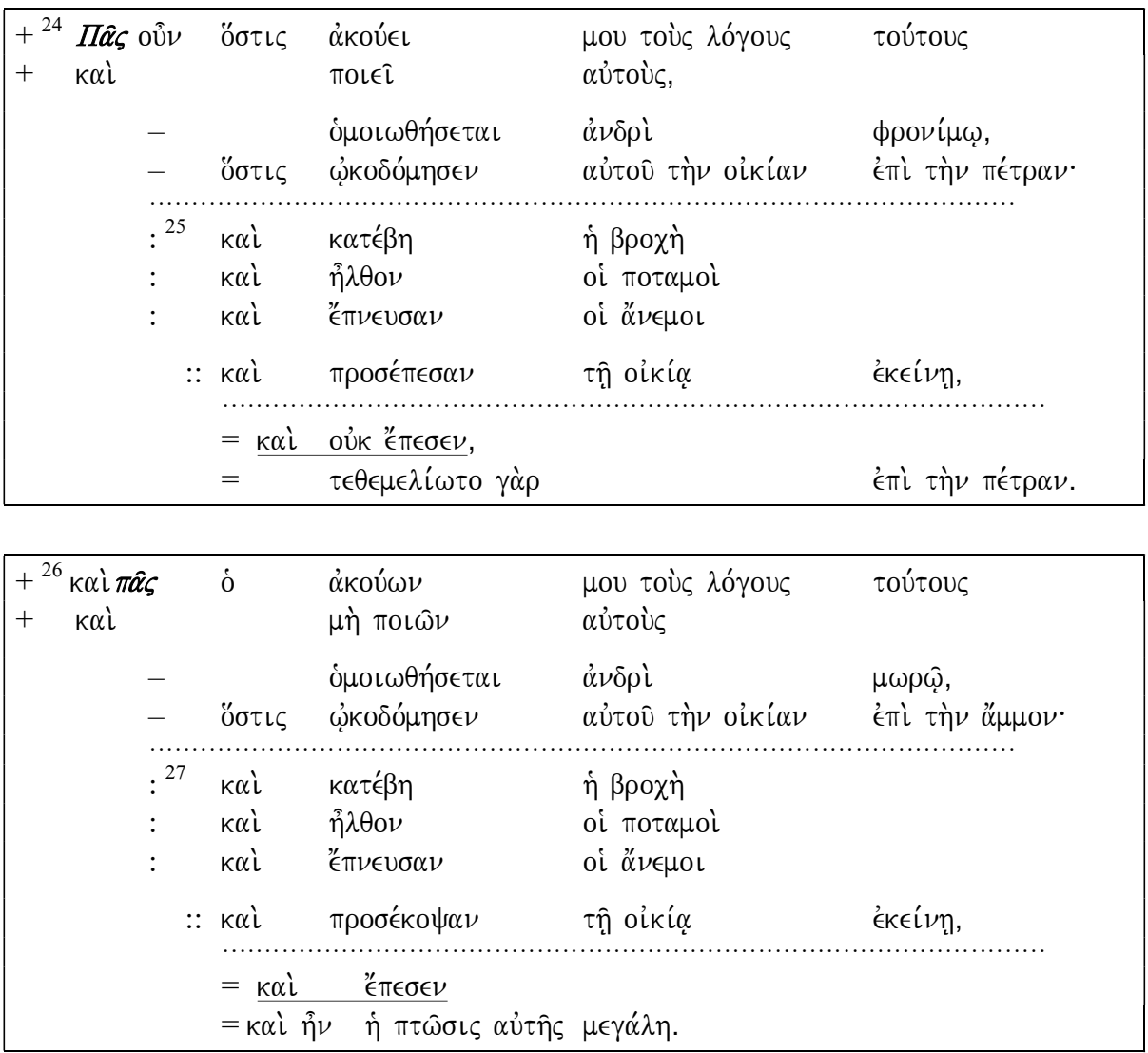

${ }_{13}$ JEBB, J., Sacred Literature, p. 213. 


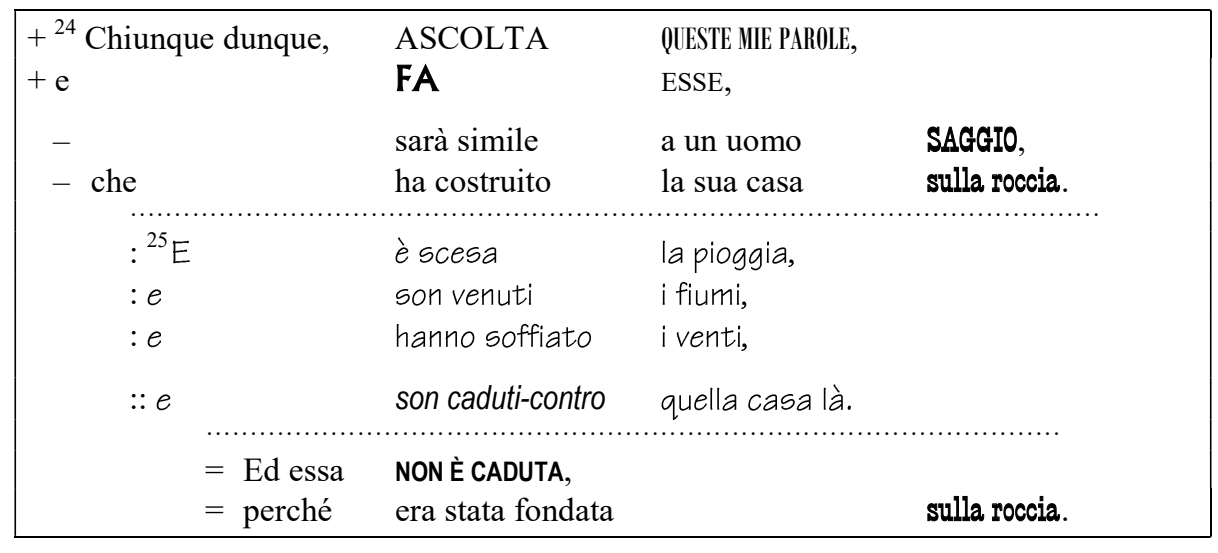

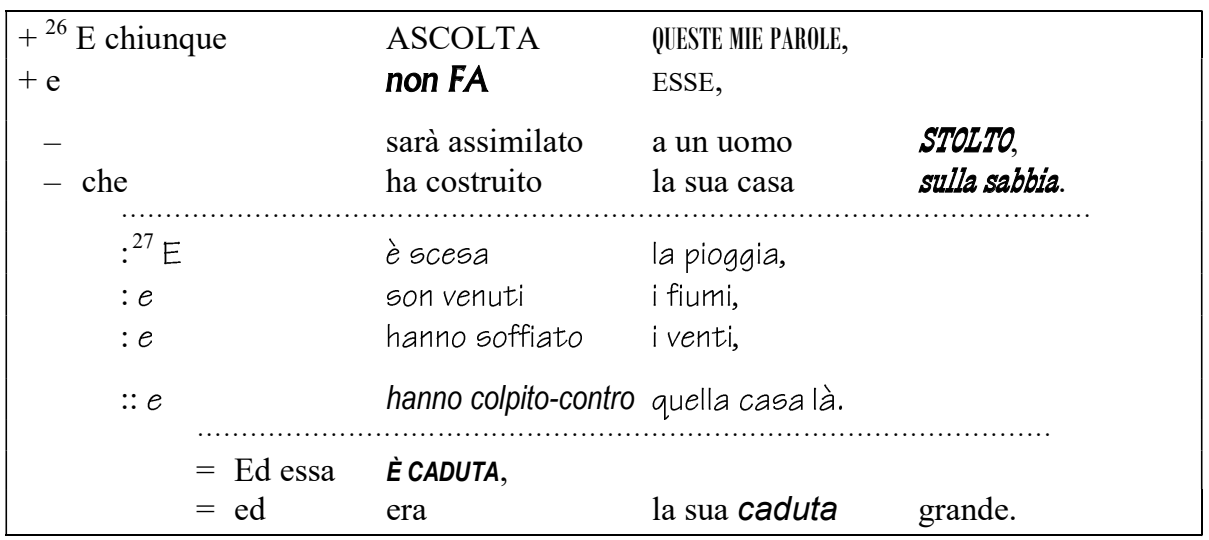

Questo testo ha le dimensioni di un "passo"; esso comprende due "parti" parallele tra di loro: ciascuna è collocata in una cornice. Ogni parte è composta da tre brani: i brani sono separati l'uno dall'altro da una linea punteggiata. La riscrittura serve a visualizzare la composizione del testo ai suoi diversi livelli di organizzazione: i membri in un'unica linea, i segmenti separati da una linea bianca, i brani separati da una linea punteggiata, le parti messe in una cornice. E perché tutto questo lavoro? Per far vedere la logica del testo. I primi due segmenti dei primi brani nel versetto 24 stabiliscono un confronto tra colui che ascolta e fa le parole di Gesù e la costruzione di una casa sulla roccia; e poi nel versetto 26 tra colui che non fa e costruisce sulla sabbia. I secondi brani includono un trimembro e un unimembro, il primo che descrive la tempesta ("pioggia", "fiumi" e "venti") e il secondo il suo colpire contro la casa. Infine gli ultimi brani comprendono un solo segmento bimembro che 
dice le conseguenze opposte della tempesta per i due tipi di case: la prima è caduta, l'altra no. Si noti che alla fine della prima parte, nell'ultimo membro è detto il motivo per cui la casa non è caduta, mentre alla fine del passo l'ultimo membro sottolinea il risultato della caduta, che è totale.

Altri testi sono composti in modo concentrico. Ecco una "parte" del Sal $115::^{14}$

\begin{tabular}{|c|c|c|c|}
\hline \multicolumn{2}{|c|}{$\begin{array}{c}+{ }^{4}{ }^{4} \text { 'ășabbêhem } \\
- \text { - MA'ǍŚŚ }\end{array}$} & \multirow{2}{*}{$\begin{array}{l}\text { kesep } \\
\text { YEDE } \\
\ldots \ldots \ldots \ldots \ldots . . . \\
\text { lāhem } \\
\text { lāhem } \\
\text { lāhem }\end{array}$} & \multirow{2}{*}{ 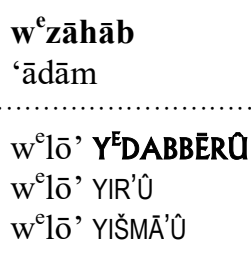 } \\
\hline $\begin{array}{l}5 \\
: \\
:\end{array}$ & $\begin{array}{l}\text { PEH- } \\
\text { 'ÊNAYIM } \\
\text { 'OZNAYIM }\end{array}$ & & \\
\hline & 'AP & lāhem & $\mathbf{w}^{\mathrm{e}} \mid \overline{\mathbf{o}}, \boldsymbol{Y}^{E} \boldsymbol{R} \hat{I} \boldsymbol{H} \hat{U} N$ \\
\hline$:^{7}$ & $\begin{array}{l}Y E D \hat{E}- \\
R A G L \hat{E}- \\
\text { ló'-YEHC }^{-}\end{array}$ & $\begin{array}{l}\text { hem } \\
\text { hem } \\
\text { GÛ }\end{array}$ & 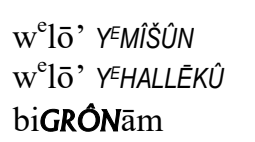 \\
\hline $\begin{array}{l}-{ }^{8} \mathrm{k}^{\mathrm{e}} \mathrm{môh} \\
\mathrm{kōl} \text { 'ăšer- }\end{array}$ & & $\begin{array}{l}\text { yihyû } \\
\text { bōțēah }\end{array}$ & $\begin{array}{l}\text { 'ŌŚÊHEM } \\
\text { bāhem }\end{array}$ \\
\hline
\end{tabular}

\begin{tabular}{|c|c|c|}
\hline $\begin{array}{c}+{ }^{4} \text { I loro idoli } \\
\quad \text { FATTI }\end{array}$ & $\begin{array}{l}\text { argento } \\
\text { da mani }\end{array}$ & $\begin{array}{l}\text { e oro } \\
\text { d'uomo. }\end{array}$ \\
\hline $\begin{array}{r}{ }^{5} \quad \text { Una } \\
: \text { degli } \\
:^{6} \text { degli }\end{array}$ & $\begin{array}{ll}\text { BOCCA } & \text { a essi } \\
\text { OCCHI } & \text { a essi } \\
\text { ORECCHI a essi }\end{array}$ & $\begin{array}{ll}\text { e non } & \text { PARLANO, } \\
\text { e non } & \text { VEDONO, } \\
\text { e non } & \text { SENTONO; }\end{array}$ \\
\hline un & NASO loro & e non ODORANO; \\
\hline $\begin{array}{c}:^{7} \text { le } \\
: \quad i \\
\text { non }\end{array}$ & $\begin{array}{l}\text { MANI di essi } \\
\text { PIEDI di essi } \\
\text { MORMORANO }\end{array}$ & $\begin{array}{l}\text { e non TOCCANO, } \\
\text { e non CAMMINANO, } \\
\text { con la GOLA di loro. }\end{array}$ \\
\hline $\begin{array}{l}-{ }^{8} \text { Come essi } \\
\text { - tutti quelli che }\end{array}$ & $\begin{array}{l}\text { saranno } \\
\text { confidano }\end{array}$ & $\begin{array}{l}\text { CHI LI FA, } \\
\text { in essi }\end{array}$ \\
\hline
\end{tabular}

(Sal 115,4-8).

${ }_{14}$ Jebb aveva notato la costruzione concentrica di Sal 135,15-18 (JEBB, J., Sacred Literature, p. 57-58); la composizione di Sal 115,4-8, simile ma più sviluppato, è stata scoperta da LUND, N. W., Chiasmus in the New Testament, p. 104-106. 
I brani estremi si corrispondono: l'ultima parola, "loro", si riferisce ai primi, "i loro idoli", cioè gli idoli delle nazioni pagane; allo stesso modo "confidano" richiama "argento e oro" perché è in queste ricchezze che molte persone ripongono la loro fiducia, "confidano". Il verbo "fare" torna nel secondo membro del v. 4 e nel primo membro del v. 8 . I due segmenti si corrispondono in modo speculare.

Mentre i brani estremi sono formati da un singolo segmento, il brano centrale ne ha tre, due trimembri che incorniciano un unimembro. In questo brano centrale anche gli organi degli idoli sono organizzati in modo concentrico: alle estremità "bocca" e "gola", ma che "non parlano" o "mormorano", poi ci sono le tradizionali coppie di "occhi" e "orecchie", "mani" e "piedi", e infine al centro, il "naso". Si noti che gli organi sono sette, cifra biblica della totalità.

Dividere questo testo in quattro strofe di quattro membri, come nella traduzione liturgica italiana, è imporgli una struttura di tipo occidentale. Innanzitutto è non rispettare i limiti di un'unità molto compatta; una tale organizzazione non è quella del testo; la "sfigura" perché non rispetta la sua composizione, la sua "figura":

\author{
${ }^{3}$ Il nostro Dio è nei cieli: \\ tutto ciò che vuole, egli lo compie. \\ ${ }^{4}$ I loro idoli sono argento e oro, \\ opera delle mani dell'uomo. \\ ${ }^{5}$ Hanno bocca e non parlano, \\ hanno occhi e non vedono, \\ ${ }^{6}$ hanno orecchi e non odono, \\ hanno narici e non odorano.
}

Gli idoli hanno tutti gli organi di un essere vivente, ma nessuno funziona: tutto è morto. Ed è quello che accadrà a quelli che li adorano: saranno morti come loro. Ci si potrà chiedere cosa viene a fare "il naso" proprio al centro! Si può pensare che sia l'unico organo che non è doppio, così come le mani e i piedi, gli occhi e le orecchie. Ma questa è una spiegazione molto superficiale! Anche la "bocca - gola" è posta in un posto di rilievo, alle estremità, e la ripetizione enfatizza la sua importanza: un dio che non parla, che non ha nulla da dire, è un dio povero, non è nulla! 
Per quanto riguarda il "naso", bisogna ricordare che, secondo il racconto del secondo capitolo della Genesi, Dio ha soffiato nel naso di Adamo un alito di vita (Gen 2,7). La fabbricazione di un idolo che non ha alito in naso, è una caricatura della creazione... Un dio che ha una bocca da cui non esce una parola, un naso in cui non entra il profumo delle offerte fatte a lui, profumi o olocausti - vale a dire animali consumati interamente dal fuoco, e il cui odore gradevole sale verso il Dio del cielo - questo è un dio assolutamente inesistente. E anche coloro che fabbricano tali idoli e li venerano sono inesistenti, non sono vere creature del Dio vivente. Ecco dunque alcuni pensieri che la composizione concentrica di questo testo invitava a produrre.

\section{Qualche esercizio}

E adesso, tocca a voi mettere le mani nella pasta: vi propongo di riscrivere un testo come avete visto che ho fatto.

5.1. Primo esercizio. Iniziamo con un breve testo della Bibbia ebraica:

4 'attâ șiwwîtâ piqqudèkā, lišmōr m ${ }^{\mathrm{e}}$ ōd :

5 'ahăălay yikkōnû derākāy, lišmōr hụqqèkā :

${ }^{4} \mathrm{Tu}$, hai comandato i tuo precetti, per osservare totalmente:

${ }^{5}$ Possano essere stabilite le mie vie, per osservare i tuoi decreti.

Richiamo delle regole di riscrittura:

- un membro per riga,

- una riga bianca fra i segmenti,

- termini allineati verticalmente per far vedere il ritmo.

Vedi la soluzione alla fine dell'articolo.

5.2. Secondo esercizio. Un altro piccolo esercizio, appena più lungo:

1 'ašrê temîmê-dārek; hahōlkîm betôrat yhwh :

2 'ašrê nōṣrê 'ēdōtāyw, b'kol-lēb yidrršûhû :

3 'ap lō'-pā'ălû 'awlâ ; bidrākāyw hālākû : 
${ }^{1}$ Beati i perfetti di via, i camminanti nella legge del Signore:

${ }^{2}$ Beati gli osservanti i suoi ordini, con tutto il cuore lo cercano:

${ }^{3}$ Mai non hanno commesso l'iniquità, nelle sue vie camminano.

Vedi la soluzione alla fine dell'articolo.

5.3. Terzo esercizio. Ancora uno, perché possiate verificare i vostri progressi:

6 'āz lō'-'ēbôš b’habbîṭ̂î, 'el-kol-mișwōtèkā :

7 'ôdkā beyōšer lēbāb ; belomdî mišp ptệ șidqekā :

8 'et-ḥuqqèkā 'ešmōr ; 'al-ta'azbēnî 'ad-m'ôd :

${ }^{6}$ Allora non mi vergognerò, guardando verso tutti i tuoi comandamenti:

${ }^{7}$ Ti loderò in rettitudine di cuore, istruito dei giudizi della tua giustizia:

${ }^{8}$ I tuoi decreti osserverò, non abbandonarmi completamente:

Soluzione alla fine dell'articolo.

\section{Funzione dell'analisi retorica biblica}

All'inizio abbiamo detto che lo scopo della retorica classica, greco-latina era di convincere, di piacere per convincere. Ora dobbiamo dire qual è la funzione della retorica biblica e semitica. La sua funzione è interrogare il lettore, per spingerlo a pensare e trovare la risposta da solo. La retorica biblica è la retorica dell'enigma. "Il greco dimostra, l'ebreo mostra". L'ebreo non cerca di convincere, di sedurre per portare il lettore ad adottare il suo punto di vista; mostra, indica la via in cui il lettore potrebbe andare se volesse capire: "Se vuoi capire, potresti andare in questa direzione".

Ecco un esempio, che permetterà di sperimentare questo tipo di retorica secondo la definizione appena enunciata. Il Salmo 113 ha tre parti, incorniciate dallo stesso "Alleluia", tradotto letteralmente come "Lodate Yah". ${ }^{15}$

${ }^{15}$ MEYNET, R., Le Psautier, p. 101-109. 


\section{${ }^{1}$ Lodate-YAH}

\begin{tabular}{|c|c|c|}
\hline $\begin{array}{l}\text { + lodate } \\
+ \text { lodate }\end{array}$ & $\begin{array}{l}\text { SERVITORI } \\
\text { il nome }\end{array}$ & $\begin{array}{l}\text { di YHWH } \\
\text { di YHWH }\end{array}$ \\
\hline $\begin{array}{l}:{ }^{2} \text { sia } \\
:: \text { lodato }\end{array}$ & $\begin{array}{l}\text { il nome di YHWH } \\
\text { - da ora } \\
\text { - }{ }^{3} \text { dal sorgere del sole } \\
\text { il nome di YHWH }\end{array}$ & $\begin{array}{l}\text { benedetto } \\
\text { fino a sempre } \\
\text { fino al suo ponente }\end{array}$ \\
\hline $\begin{array}{l}+{ }^{4} \text { ESALtato } \\
+\end{array}$ & $\begin{array}{l}\text { al di sopra di TUTTE LE NAZIONI } \\
\text { al di sopra dei CIELI }\end{array}$ & $\begin{array}{l}\text { YHWH } \\
\text { LA SUA GLORIA }\end{array}$ \\
\hline
\end{tabular}



Lodate-YAH.

Nella prima parte (1b-4) notiamo solo la costruzione speculare del brano centrale (2-3). Alle estremità due auguri sinonimi: "sia il nome di Yhwh benedetto" e "lodato (sia) il nome di Yhwh". Tra i due auguri, il complemento di tempo che indica la totalità ("da ora fino a sempre"), quindi il complemento di luogo che indica anche la totalità ("dal sorgere del sole fino al suo ponente").

Al centro, una domanda, l'unica del salmo. Tutti gli articoli consultati su questo salmo dicono che si tratta di una "domanda retorica", cioè di una falsa domanda, la cui risposta è ovvia: "non c'è nessuno come il Signore Dio nostro". Questa risposta non è sbagliata, ovviamente, ma è l'unica risposta? È questa la più importante? Bisogna esaminare i rapporti tra le parti e prestare molta attenzione alle parole identiche. $\mathrm{E}$ anche ai sinonimi: si può notare che il primo segmento della terza parte (5b-6) corrisponde all'ultimo segmento della prima parte (4): alla 
coppia "i cieli e la terra" di 6 corrisponde quella delle "nazioni" e dei "cieli". Inoltre, le prime parole di questi segmenti, "Esaltato" e "Si alza", sono sinonimi.

Una parola è ripetuta identicamente nelle due parti principali del testo: "Esaltato" in 4 ed "esalta" in 7. La terza parte fornisce la risposta alla domanda centrale. Quale altro dio ha fatto o farebbe ciò che ha fatto Yhwh per gli uomini? Qual è il dio, la cui trascendenza assoluta, come descritta alla fine della seconda parte (4), consiste nell'"abbassarsi" fino alla "polvere" et all'"immondizia", dove giacciono lo "sprovveduto" e "il povero"? Non solo il Signore si abbassa al più basso; se lo fa, è per "esaltare" l'umiliato (7) come egli stesso è "esaltato" sopra tutto (4). Quindi la risposta alla domanda che sembra ovvia - "Non c'è assolutamente nessuno sulla terra e in cielo che è come il Signore nostro Dio!" (vedi Sal 40,6; 86,8) - può essere completata o addirittura completamente rovesciata: sono i poveri e gli sterili che sono "come il Signore nostro Dio". È davvero fantastico. Eppure, questo è ciò a cui l'uomo era stato invitato sin dall'origine: "Dio creò l'uomo a sua immagine, a immagine di Dio lo creò; uomo e donna li creò" (Gen 1,27).

Ecco ciò a cui serve l'analisi retorica biblica: a meglio capire il testo, nella sua profondità.

Ma non fermiamoci a questo punto e andiamo un po' oltre. Il Sal 113 è il primo di un insieme che riunisce i sei salmi dell'Hallel egiziano (Sal 113-118); l'Hallel è cantato dagli ebrei e dai cristiani durante la celebrazione della notte di Pasqua. I salmi 113 e 114 formano una sequenza e, se possiamo ovviamente leggerli separatamente, è ancora più significativo leggerli insieme:

I due salmi hanno in comune il nome "Dio" $(113,5 \mathrm{a} ; 114,7)$, "terra" nelle ultime parti $(113,6 ; 114,7)$, "figli" $(113,9 ; 114,4.6)$ e "casa" le cui due occorrenze svolgono il ruolo di termini mediani $(113,9 ; 114,1)$.

"Egitto", il "popolo barbaro", vale a dire straniero $(114,1)$, è una tra "tutte le nazioni" $(113,4)$.

I due salmi terminano con un capovolgimento: dal "debole" sollevato dalla "polvere" fino alla "sterile" che diventa "madre di figli felice" nel primo salmo $(113,7-9)$; "la rupe" è cambiata "in lago d'acqua", "la roccia in sorgenti d'acqua" nel secondo salmo (114,7-8), in entrambi i casi grazie all'intervento di Dio. 
$113,{ }^{1}$ Lodate Yah. Lodate, servi del Signore, lodate il nome del Signore. ${ }^{2}$ Sia benedetto il nome del Signore, da ora e fino a sempre; ${ }^{3}$ dal sorgere del sole fino al suo tramonto sia lodato il nome del Signore. ${ }^{4}$ Esaltato sopra tutte le nazioni il Signore, sopra i cieli la sua gloria.

${ }^{5}$ Chi è come il Signore, nostro DIO?

Si alza per sedere, ${ }^{6}$ si abbassa per vedere sui cieli e sulla TERRA. ${ }^{7}$ Solleva dalla polvere il debole, dall'immondizia esalta il povero, ${ }^{8}$ per farlo-sedere tra i prìncipi, tra i prìncipi del suo popolo; ${ }^{9}$ fa-sedere la sterile di casa, madre di FIGLI felice. Lodate Yah.

$114,{ }^{1}$ Quando Israele uscì dall'Egitto, la casa di Giacobbe da un popolo barbaro, ${ }^{2}$ Giuda divenne il suo santuario, Israele il suo dominio.

${ }^{3}$ Il mare vide e si ritrasse, il Giordano si volse indietro, ${ }^{4}$ le montagne saltellarono come arieti, le colline come FIGLI di un gregge. ${ }^{5}$ Che hai tu, mare, per fuggire, e tu, Giordano, per volgerti indietro? ${ }^{6}$ Perché voi, montagne, saltellate come arieti e voi, colline, come FIGLI di un gregge?

${ }^{7}$ Trema, o TERRA, davanti al Signore, davanti al DIO di Giacobbe, ${ }^{8}$ che muta la rupe in un lago d'acqua, la roccia in sorgenti d'acqua.

La domanda centrale del Sal 113 richiama quella sulla quale è focalizzato il Canto del Mare:

Chi è come te fra gli déi

fra gli déi, Signore?

Chi è come te, maestoso in santità, terribile nelle imprese, autore di prodigi? (Es 15,11)

Ora il Sal 114 celebra questo evento fondatore del popolo di Israele; non solo l'uscita dall'Egitto all'inizio dell'esodo, ma anche la traversata del Giordano che segna la sua fine e l'ingresso nella terra promessa. Quindi a questo tipo di merismo che dice l'azione di Dio corrispondono gli altri due merismi dell'inizio del Sal 113: "da ora e fino a sempre", "dal sorgere del sole fino al suo tramonto" che caratterizzano la lode degli uomini $(113,2-3)$, così come quello con cui termina la prima parte e il cui soggetto è di nuovo il Signore: "sopra tutte le nazioni $[\ldots]$ sopra i cieli" $(113,4)$. 
Il rapporto di senso tra i due salmi è lungi dall'essere evidente a prima vista. Eppure, non appena abbiamo compreso il legame tra i rispettivi centri del Sal 113 e del Cantico del mare, tutto si illumina. L'uscita dall'Egitto, l'intero percorso dell'esodo che porterà al "santuario" di Giuda (Sal 113,2; Es 15,17), rappresenta il caso più emblematico dell'azione salvifica di Dio. Strappando i figli di Israele dalla schiavitù nella terra d'Egitto, il Signore "esalta il povero" $(113,7)$ ed è così che manifesta "la sua gloria" "al di sopra di tutte le nazioni" $(113,4)$ e che "la terra" tremerà "davanti al Maestro [...] davanti al Dio di Giacobbe" (Sal 114,7). Perciò chi viene esaltato dal Signore esalta il suo salvatore in cambio. Gli schiavi nel paese d'Egitto son diventati "servi del Signore" (Sal 113,1). ${ }^{16}$

\section{Ancora qualche esercizio}

I "brani" che avete già riscritto formano insieme il primo passo del Sal 119, il grande salmo della Legge.

Quarto esercizio. Ora potere riscriverlo in tre cornici (come il Sal 113 è stato riscritto in tre cornici). Adesso tuttavia non dovete più evidenziare le relazioni interne di ciascuna delle tre parti come avete fatto finora, ma evidenziate solo le relazioni tra le tre parti.

Vedi la soluzione dell'esercizio alla fine dell'articolo.

\section{L'interpretazione}

A che cosa serve tutto questo lavoro tecnico, molto lungo, molto impegnativo? Semplicemente a capire meglio il testo. Questo è l'obiettivo di ogni lavoro esegetico.

In questo caso, ecco alcuni punti di interpretazione:

\subsection{Beato!}

Come l'intero Salterio, il Sal 119 inizia proclamando "beati" coloro che, evitando "l'iniquità", si comportano secondo la legge del Signore (Sal 1). Prima di entrare in scena, di parlare di se stesso, e prima di rivolgersi a Dio, il salmista elogia coloro che custodiscono le sue testimonianze e le cercano. Come se avesse bisogno di modelli da seguire, esempi da imitare, guide per tracciare la via.

${ }^{16}$ MEYNET, R., Le Psautier, p. 121-122. 


\subsection{Sulla stessa via}

Coloro le cui vie sono perfette sono quelli che camminano nelle vie di Dio, sui sentieri tracciati dalla sua legge (1-2). Il più caro desiderio del salmista, il primo che emette, è che le sue vie siano stabilite secondo i decreti del suo Dio. I sentieri dell'uomo giusto e quelli del Signore s'incontrano. Più esattamente, coincidono, sono le stesse strade, come se Dio e i suoi fedeli camminassero insieme. "Uomo, ti è stato insegnato ciò che è buono e ciò che richiede il Signore da te: praticare la giustizia, amare la bontà, camminare umilmente con il tuo Dio" (Mi 6,8).

\subsection{Un affare di cuore}

"La legge" di Dio, i suoi "precetti”, i suoi “ordini”, i suoi "giudizi”, tutte queste parole sono pronunciate per essere attuate, per mettere in moto l'uomo invitato a seguire la via del suo Signore. Tuttavia, i suoi piedi non potrebbero muoversi se non ci fosse il "cuore". È infatti "con tutto il cuore" che i giusti lo cercano (2), il cuore essendo la sede dell'intelligenza, della volontà e anche dell'amore. È così che il salmista anch'esso promette al suo Signore di lodarlo "nella rettitudine del cuore" (7), perché avrà ricevuto l'istruzione che cercava.

\subsection{Con l'aiuto di Dio}

Nel cuore del passo il salmista si rivolge a Dio. Di fronte all'esigenza del Signore che chiede di osservare la sua legge "completamente" (4), egli spera di essere in grado di rispondere a tale invito e il desiderio che esprime dinanzi al suo Dio (5) suona come una richiesta di assistenza e supporto. Continua la sua preghiera, sperando di essere all'altezza della sua chiamata osservando "tutti gli ordini” del Signore. Ma alla fine, come se temesse di non poterlo fare, la sua supplica è alquanto angosciata quando chiede a Dio di non "abbandonarlo completamente" (8). 


\section{Un'intera sequenza}

Adesso passiamo al livello superiore a quello del "passo": studieremo una "sequenza" che include diversi passi in un tutto unificato, organico. Si tratta della sequenza centrale del Discorso sul monte in Mt 5-7 (Mt 6,1-18). ${ }^{17}$

\subsection{Il primo passo: l'introduzione $(6,1)$}

Un solo versetto introduce l'insieme:

\begin{tabular}{|c|c|c|c|}
\hline 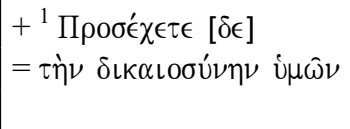 & 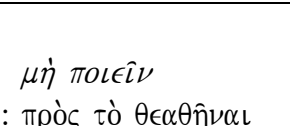 & $\epsilon \mu \pi \rho 0 \sigma \theta \epsilon \nu$ & $\begin{array}{l}\tau \hat{\omega} \nu \dot{\alpha} \nu \theta \rho \omega \dot{\omega} \pi \omega \nu \\
\alpha \dot{U} \tau o \hat{\iota} \varsigma .\end{array}$ \\
\hline $\begin{array}{l}+\epsilon \hat{l} \delta \dot{\epsilon} \mu \eta^{\prime} \gamma \epsilon, \\
=\mu \iota \sigma \theta \dot{\mathrm{o} \nu}\end{array}$ & 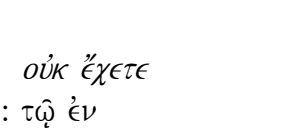 & $\underline{\pi \alpha \rho \dot{\alpha}}$ & 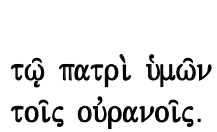 \\
\hline $\begin{array}{l}+{ }^{1} \text { State-attenti } \\
\quad=\text { la vostra giustizia }\end{array}$ & $\begin{array}{l}\text { di non praticare } \\
\text { : per essere ammirati }\end{array}$ & davanti & $\begin{array}{l}\text { agli uomini } \\
\text { da loro; }\end{array}$ \\
\hline $\begin{array}{l}+ \text { se no } \\
\quad=\text { ricompensa }\end{array}$ & $\begin{aligned} & \text { non avrete } \\
& \text { : quello }\end{aligned}$ & presso & $\begin{array}{l}\text { il vostro Padre } \\
\text { nei cieli. }\end{array}$ \\
\hline
\end{tabular}

Diciamo solo che questo breve passo mette in parallelo da una parte "la vostra giustizia" e il suo "salario" o la sua "ricompensa" e dall'altra parte gli "uomini" e "il vostro Padre".

\footnotetext{
${ }^{17}$ MEYNET, R., Traité de rhétorique biblique, p. 371; vedi soprattutto la tesi (sotto la mia direzione) di LORI, G. Il discorso della montagna, dono del Padre (Mt 5,1-8,1). Le riscritture sono mie.
} 


\subsection{Il terzo passo $(6,5-6)$}

Il passo seguente è di composizione analoga:

\begin{tabular}{|c|c|c|}
\hline \multicolumn{3}{|c|}{. $5 \mathrm{~K} \alpha \mathrm{i} \quad$ ó $\alpha \alpha \nu \pi \rho 0 \sigma \epsilon u ́ \chi \eta \sigma \theta \epsilon$} \\
\hline 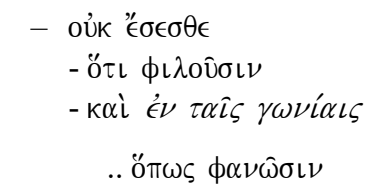 & 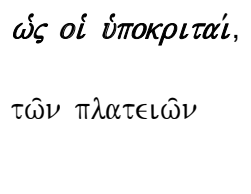 & 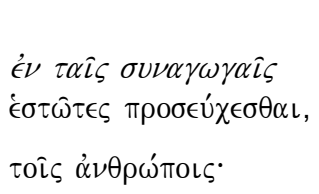 \\
\hline $\begin{array}{l}=\dot{\alpha} \mu \grave{\eta} \nu \\
=\dot{\alpha} \pi \dot{\epsilon} \chi O v \sigma \iota \nu\end{array}$ & $\begin{array}{l}\lambda \epsilon^{\prime} \gamma \omega \\
\tau \grave{\nu} \nu \mu \iota \sigma \theta \grave{\nu} \nu\end{array}$ & 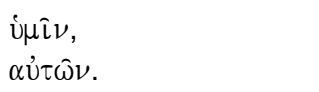 \\
\hline
\end{tabular}

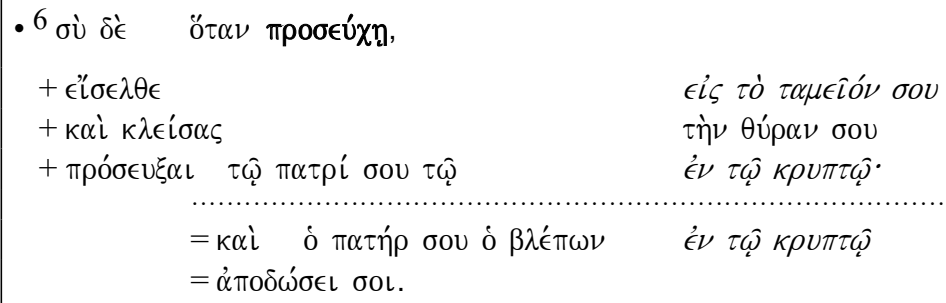

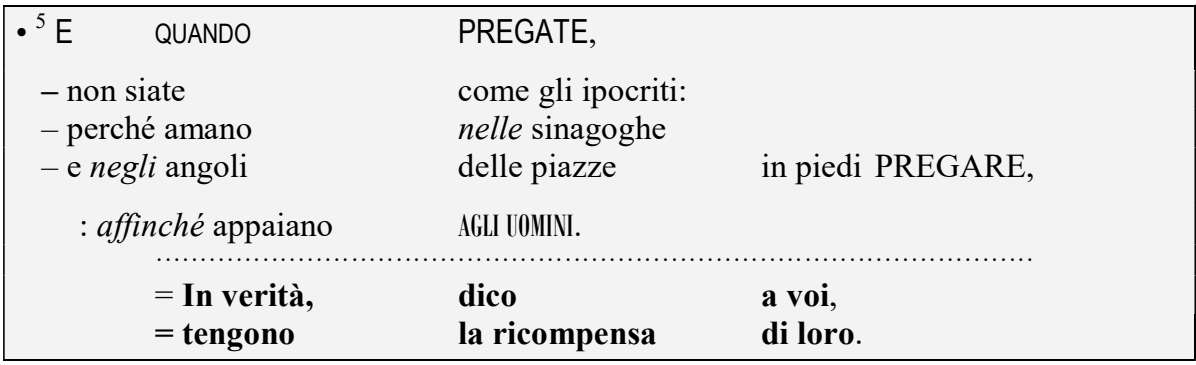

\begin{tabular}{|c|c|c|}
\hline $\begin{array}{l}\text { - }{ }^{6} \text { MA TU, QUANDO } \\
+ \text { entra } \\
\text { + e chiusa } \\
\text { + PREGA }\end{array}$ & $\begin{array}{l}\text { PREGHI, } \\
\text { nella tua camera, } \\
\text { la tua porta, } \\
\text { il tuo Padre }\end{array}$ & che (è) nel segreto. \\
\hline $\begin{array}{l}=\mathbf{E} \text { IL TU0 PADRE } \\
=\text { ridarà in cambio }\end{array}$ & $\begin{array}{l}\text { che vede } \\
\text { a te. }\end{array}$ & nel segreto \\
\hline
\end{tabular}




\subsection{L'ultimo passo (16-18)}

Quinto esercizio. Adesso fate la riscrittura dell'ultimo passo:

${ }^{16}$ E quando digiunate, non assumete aria malinconica come gli ipocriti, che si sfigurano la faccia per far vedere agli uomini che digiunano. In verità vi dico: hanno già ricevuto la loro ricompensa. ${ }^{17} \mathrm{Tu}$ invece, quando digiuni, profumati la testa e lavati il volto, ${ }^{18}$ perché la gente non veda che tu digiuni, ma solo tuo Padre che è nel segreto; e il Padre tuo, che vede nel segreto, ti ricompenserà.

\subsection{L'intera sequenza (Mt 6,1-18)}

E ora, vediamo l'intera sequenza, senza entrare in tutti i dettagli, per mancanza di spazio. Si tratterà di capire i rapporti tra i passi; ognuno deve essere interpretato in sé, ma il significato del tutto è più della somma dei sensi di ciascun passo.

Le opere di "giustizia" sono tre: elemosina, preghiera, digiuno L'opera centrale, la preghiera, è particolarmente sviluppata: al passo dei versetti 5-6, che sono paralleli ai passi delle elemosine (2-4) e del digiuno (16-18), viene aggiunto un lungo passo che fornisce nuovi consigli (7-8 e 14-15), e questi nuovi consigli incorniciano la preghiera del Signore. ${ }^{18}$

Per l'interpretazione dell'intera sequenza, si tratta di capire qual è la relazione tra le tre opere di "giustizia".

La richiesta del pane non è solo la chiave di volta della preghiera del Signore, è anche la chiave di lettura dell'insieme costituito da elemosina, preghiera e digiuno. Una prima domanda potrebbe sorgere in questi termini, come quelli di un enigma: cosa c'è in comune tra l'elemosina e il digiuno? Queste due pratiche religiose devono essere correlate l'una all'altra, dal momento che sono simmetriche, che si corrispondono da ciascun lato della preghiera che incorniciano; inoltre, sono composte esattamente sullo stesso modello.

\footnotetext{
${ }^{18}$ MEYNET, R., La composition du Notre Père, p. 1-28; traduzione italiana, MEYNET, R., La composizione del Padre nostro. Studia Rhetorica Biblica et Semitica, n.19, p. 1-20.
} 
${ }^{1}$ State attenti a non praticare la vostra giustizia davanti agli uomini per essere ammirati da loro, altrimenti non c'è ricompensa per voi presso il Padre vostro che è nei cieli.

2 Dunque, QUANDO FAI L'ELEMOSINA, non suonare la tromba davanti a te, come fanno gli ipocriti nelle sinagoghe e nelle strade, per essere lodati dalla gente. In verità io vi dico: hanno già ricevuto la loro ricompensa.

${ }^{3}$ Invece, MENTRE TU FAI L'ELEMOSINA, non sappia la tua sinistra ciò che fa la tua destra, ${ }^{4}$ perché la tua elemosina resti nel segreto; e il Padre tuo, che vede nel segreto, ti ricompenserà.

${ }^{5}$ E QUANDO PREGATE, non siate simili agli ipocriti che, nelle sinagoghe e negli angoli delle piazze, amano pregare stando ritti, per essere visti dalla gente. In verità io vi dico: hanno già ricevuto la loro ricompensa.

6 Invece, QUANDO TU PREGHI, entra nella tua camera, chiudi la porta e prega il Padre tuo, che è nel segreto; e il Padre tuo, che vede nel segreto, ti ricompenserà.

${ }^{7}$ Pregando, non sprecate parole come i pagani: essi credono di venire ascoltati a forza di parole. ${ }^{8}$ Non siate dunque come loro, perché il Padre vostro sa di quali cose avete bisogno prima ancora che gliele chiediate. ${ }^{9}$ Voi dunque pregate così:

Padre nostro che sei nei cieli,

+ sia santificato il tuo nome,

$-{ }^{10}$ venga il tuo regno,

: sia fatta la tua volontà, come in cielo cosi in terra.

\section{1 il pane nostro quotidiano \\ da a noi oggi}

: ${ }^{12} \mathrm{e}$ rimetti a noi i nostri debiti come anche noi li rimettiamo ai nostri debitori,

$-{ }^{13}$ e non abbandonarci alla tentazione,

+ ma liberaci dal male.

14 Se voi infatti perdonerete agli altri le loro colpe, il Padre vostro che è nei cieli perdonerà anche a voi; ${ }^{15}$ ma se voi non perdonerete agli altri, neppure il Padre vostro perdonerà le vostre colpe.

${ }^{16}$ E QUANDO DIGIUNATE, non diventate malinconici come gli ipocriti, che assumono un'aria disfatta per far vedere agli altri che digiunano. In verità io vi dico: hanno già ricevuto la loro ricompensa.

17 Invece, QUANDO TU DIGIUNI, profumati la testa e làvati il volto, ${ }^{18}$ perché la gente non veda che tu digiuni, ma solo il Padre tuo, che è nel segreto; e il Padre tuo, che vede nel segreto, ti ricompenserà.

In entrambi i casi, c'è l'accettazione di una mancanza: colui che dà l'elemosina e colui che digiuna rinunciano al pane che hanno, che hanno chiesto al loro Padre nella preghiera e hanno ricevuto da lui. L'elemosina è il pane del quale ci si priva per darlo ai poveri, agli affamati. Con l'elemosina l'uomo 
compie le opere di Dio, imita la generosità del Padre suo e si manifesta così come suo figlio. Così parlava Giobbe: "Io ero il padre dei poveri" $(\mathrm{Gb} 29,16)$. Colui che sta digiunando rinuncia anche, per un certo tempo, a mangiare il suo pane. Significa così che non è l'origine di se stesso, che non è dal suo stesso lavoro che deriva il suo sostentamento, che la vita non gli viene dal pane, ma da Colui che gli dà il pane; in altre parole, davanti a Dio — davanti a Dio solo, dice Gesù - riconosce la sua filiazione. In breve, praticare l'elemosina è essere un padre, praticare il digiuno significa comportarsi da figlio. "Praticare la giustizia" significa quindi trovare il suo giusto posto sulla linea della filiazione, quella che si riceve e quella che si dà; come il pane che lo simboleggia.

\section{Un'altra sequenza (Mt 5,3-16)}

L'analisi di un altro testo (Mt 5,3-16), sempre nel Discorso sulla montagna, ci riserva una bella sorpresa. ${ }^{19}$

Si dice spesso che le Beatitudini sono otto perché il versetto 10 fa "inclusione" con il versetto 3. È vero che i loro secondi membri sono identici ("perché a essi è il regno dei cieli"). Ma due frasi ripetute possono avere una funzione diversa da quella di "termini estremi", cioè, che segnano i limiti di un'unità letteraria. Possono anche svolgere il ruolo di "termini iniziali", vale a dire che segnalano l'inizio di due unità diverse. È questo il caso. Inoltre, se le prime sette beatitudini sono brevi (ciascuna è un segmento bimembro), l'ottavo, quello della persecuzione è molto più sviluppato (10-12). La sua importanza è sottolineata dal posto centrale che occupa al centro della sequenza.

Non si tratta qui di eseguire un' analisi dettagliata della sequenza, passo dopo passo, quindi dell'intera sequenza. Bisognava solo osservare che il primo passo (3-9) comprende sette beatitudini come il Padre Nostro include sette richieste; osservare anche che la beatitudine centrale (6) corrisponde alla domanda centrale del Padre nostro: al centro della preghiera del Signore chiediamo il nostro pane, al centro delle beatitudini sono saziati coloro che hanno fame e sete della giustizia. Quindi capiamo che "il pane" ha qualcosa a che fare con la "giustizia". Ma che cos'è questa "giustizia"? Il resto della sequenza lo dirà. Infatti, la parola "giustizia" è ripresa all'inizio del secondo passo (10a): la giustizia, è ciò "a causa di cui" i discepoli saranno perseguitati. E nel seguente versetto impariamo che sono perseguitati "a causa di"

${ }^{19}$ MEYNET, R., I frutti dell'analisi retorica, p. 403-436. 
Gesù. La "giustizia" è Lui! Quando interpretiamo il pane della preghiera del Signore come il corpo di Cristo, non siamo lontani dall'identificare la giustizia con Lui ...

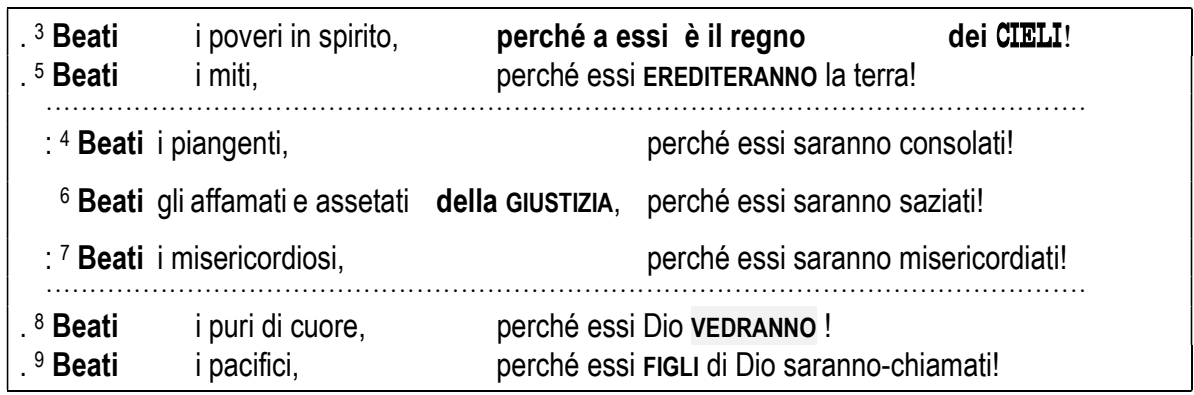

10 Beati i perseguitati a causa de la GIUSTIZIA, perché a essi è il regno dei CIELI!

${ }^{11}$ Beati siete quando insulteranno voi e perseguiteranno e diranno ogni male contro di voi a causa di ME.

12 Rallegratevi ed esultate,

perché il vostro salario è grande nei CIELI!

Così infatti perseguitarono i profeti prima di voi.

\begin{tabular}{|c|c|c|c|}
\hline \multicolumn{4}{|c|}{+13 Voi, siete IL SALE $\quad$ della terra. } \\
\hline \multicolumn{2}{|c|}{.. Ma se il sale perdesse il sapore, } & \multicolumn{2}{|r|}{ con che cosa lo si salerà? } \\
\hline & $\begin{array}{l}=A \text { niente serve } \\
=\end{array}$ & \multicolumn{2}{|r|}{$\begin{array}{l}\text { che di essere gettato fuori } \\
\text { per essere calpestato dagli uomini. }\end{array}$} \\
\hline \multicolumn{4}{|c|}{ + 14 Voi, siete LA LUCE del mondo. } \\
\hline \multicolumn{2}{|c|}{$\begin{array}{l}\text { - Non può una città essere nascosta } \\
::{ }^{15} \text { e non accendono una lucerna } \\
\text { :: ma sopra il lucerniere }\end{array}$} & \multicolumn{2}{|r|}{$\begin{array}{l}\text { sopra un monte collocata. } \\
\text { e la mettono sotto il moggio } \\
\text { e risplenda per tutti quelli nella casa. }\end{array}$} \\
\hline \multicolumn{3}{|c|}{$\begin{array}{l}=16 \text { Cosi risplenda la luce } \\
\text { Perché VEDANO le } \\
=\text { e glorifichino IL PADRE }\end{array}$} & $\begin{array}{l}\text { vostra davanti agli uomini } \\
\text { vostre buone opere } \\
\text { vostro quello nei CIELI. }\end{array}$ \\
\hline
\end{tabular}




\section{Conclusione: il Padre nostro e una preghiera musulmana}

Non si può riprendere in questa sede l'analisi dettagliata della Preghiera del Signore; ${ }^{20}$ l'abbiamo appena vista nel suo contesto immediato. Analizzeremo un testo musulmano per terminare la breve esposizione. È un testo che alcuni chiamano il "Padre nostro musulmano" perché è molto simile alla preghiera del Signore, ma con notevoli differenze.

hadith al-ruqya (hadîth per la guarigione) IBN HANBAL, Musnad, $\mathrm{n}^{\circ} 23839$ (con qualche variante in ABÛ-DÂWUD, Sunan, Libro 22, Tibb, 19 bâb kayfa al-ruqy)

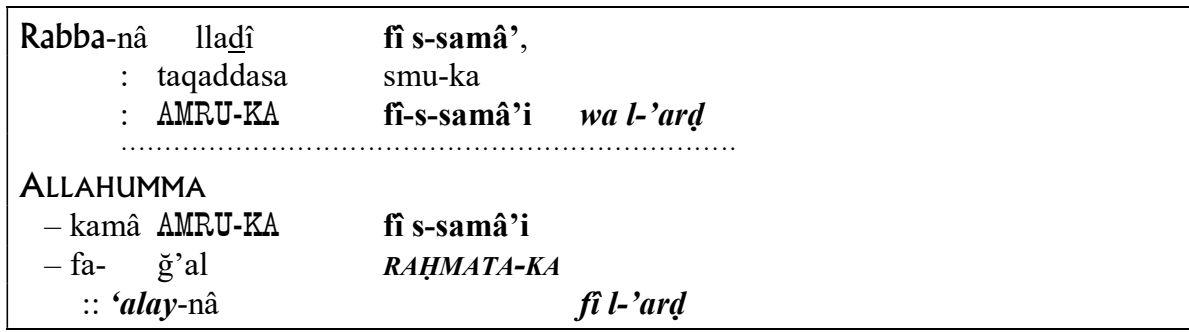

\begin{tabular}{|c|c|c|}
\hline ALLAI & MMA & rabba t-tayyibîna \\
\hline- & gfir la-nâ & hûba-nâ \\
\hline- wa & dunûba-nâ & wa ḥațayânâ-nâ \\
\hline- wa & nazzil & RAHMATA-KA \\
\hline- wa & šifâ'an & min šifâ'i-ka \\
\hline $\begin{array}{l}:: \\
::\end{array}$ & $\begin{array}{l}\text { â mâ bi-Fulân } \\
\text { yabra' }\end{array}$ & min šakwa \\
\hline
\end{tabular}

${ }^{20}$ MEYNET, R., A Análise retórica, p. 391-408. 
...da Fadâla b. 'Ubayd al-Ansârî che ha detto: il profeta mi ha insegnato una preghiera profilattica e mi ha ordinato di usarla per chi volevo, dicendomi: "Dì:

\begin{tabular}{|c|c|c|c|}
\hline Signore nostro che (sei) & nel cielo, & & 1 \\
\hline : sia santificato & il tuo nome & & 2 \\
\hline : II TUO COMANDO (sia) & nel cielo & e la terra. & 3 \\
\hline \multicolumn{4}{|l|}{ ODDIO, } \\
\hline - come IL TUO COMANDO & (è) & nel cielo & 5 \\
\hline$-\operatorname{così~metti~}$ & la tua misericordia & & 6 \\
\hline : su di noi & & sulla terra & 7 \\
\hline
\end{tabular}

\begin{tabular}{|c|c|c|c|}
\hline ODDIO, & Signore & dei buoni, & 8 \\
\hline$-\quad p$ & perdona a noi & le nostre colpe & 9 \\
\hline$-\mathrm{e} \quad \mathrm{i}$ & i nostri peccati & e le nostre mancanze & 10 \\
\hline$-\mathrm{e} f$ & fa' scendere & la tua misericordia & 11 \\
\hline$-\mathrm{e} \quad \mathrm{u}$ & una guarigione & delle tue guarigioni & 12 \\
\hline & su ciò di cui $N$. & soffre & 13 \\
\hline$:: \mathrm{e}$ & e che guarisca. & & 14 \\
\hline
\end{tabular}

I due brani della prima parte sono paralleli. Iniziano con un'apostrofe, il "comando" e la "misericordia" che viene aggiunta in seguito devono essere realizzati "in cielo" e "sulla terra".

La seconda parte è anche introdotta da un'apostrofe: "Oddio, Signore dei buoni" corrisponde a "Signore nostro" all'inizio della prima parte. Nel primo brano (8-10), la preghiera è fatta per "noi", per ottenere il perdono dei nostri peccati, mentre il secondo pezzo richiede la guarigione per il paziente per il quale preghiamo. Notiamo la ripresa della "misericordia" da una parte all'altra (6.11).

Le somiglianze tra questa preghiera e il Padre Nostro di Mt saltano agli occhi, sin dalla prima apostrofe (linea 1). La prima richiesta (2) è identica a quella della preghiera del Signore; la seconda (3) assomiglia alla terza richiesta del Padre ("Sia fatta la tua volontà come nei cieli così sulla terra"); la richiesta di perdono si trova sulle linee 9-10. Tuttavia non basta notare le somiglianze, dobbiamo anche vedere cosa distingue questo testo dal Padre nostro. La composizione è parallela mentre quella del Padre Nostro di Matteo 
è concentrica. Questo dal punto di vista della forma. Dal punto di vista del contenuto le differenze sono essenziali. Sin dalla prima parola, colui a cui è rivolta la preghiera non è chiamato "Padre nostro" ma "Signore nostro"; tutta la differenza è già lì. Molti cristiani, così abituati a considerare Dio come Padre, non possono nemmeno immaginare che possa essere diversamente negli altri credenti. Ora l'Islam differisce dalla fede cristiana su questo punto fondamentale della filiazione divina. Per l'Islam, Gesù non è affatto il Figlio di Dio; ancor di meno i suoi discepoli! Si capisce quindi perché la richiesta centrale della Preghiera del Signore, la richiesta di pane che è la specifica richiesta del figlio, è totalmente assente dalla preghiera che la tradizione musulmana fa risalire a Maometto stesso. Si capisce anche che se questa preghiera musulmana invoca il perdono di Dio, non riprende il "come noi perdoniamo anche" della preghiera cristiana. Si sarà notato che un "come" si trova nella terza richiesta (5), alla quale corrisponde un "cosi" (6); ma questo "come" indica solo il lavoro dell'unico Dio "in cielo" e "sulla terra". Nel Padre nostro, al contrario, la volontà di Dio è affidata all'uomo perché sia adempiuta "come nei cieli e sulla terra", cioè sulla terra dagli uomini, come in cielo da Dio.

\section{Soluzioni}

\begin{tabular}{|c|c|c|}
\hline $\begin{array}{l}-{ }^{4} \text { ' attâ } \\
\quad:: \text { lišmōor }\end{array}$ &  & piqqudèkā \\
\hline $\begin{array}{l}-{ }^{5} \text { ' ahălay } \\
\quad:: \text { lišmōor } r\end{array}$ & $\begin{array}{l}\text { yikkōnû } \\
\text { huqqèkāa }\end{array}$ & derākāy \\
\hline $\begin{array}{l}-{ }^{4} \mathrm{Tu}, \\
\quad:: \text { PER OSSERVARE }\end{array}$ & $\begin{array}{l}\text { hai comandato } \\
\text { completamente. }\end{array}$ & I TUOI PRECETTI, \\
\hline $\begin{array}{l}-{ }^{5} \text { Possano } \\
\quad: . \text { PER OSSERVARE }\end{array}$ & $\begin{array}{l}\text { essere stabilite } \\
\text { I TUOI DECRETI }\end{array}$ & LE MIE VIE \\
\hline
\end{tabular}

(SAL 119,4-5).

Complementarità tra i due segmenti: il primo ha Dio come soggetto, il secondo riguarda l'uomo, cioè l'io del salmista. Anche i secondi membri sono complementari: uno è l'oggetto del desiderio di Dio ("perché siano osservati"), l'altro della volontà dell'uomo. I "precetti" di Dio diventeranno le mie "vie". 
Soluzione del primo esercizio:

$+{ }^{1}$ 'ašrê

temîmê-

DĀREK

.. HAHŌLKîM

$b^{e}$ tôrat

yhwh

$+{ }^{2}$ 'ašrê

nōṣrê

‘ēdōtāyw

.. b ${ }^{\mathrm{e}}$ kol-lēb

yidr šsuhhû

$+{ }^{3}$ ap

lō'-pā'ălû

‘awlâ

.. biDRĀKĀYW

$\boldsymbol{H} \bar{A} L \bar{A} K \hat{U}$

$+{ }^{1}$ Beati

i perfetti

di VIA,

.. I CAMMINANTI

nella legge

del Signore!

$={ }^{2}$ Beati

gli osservanti

i suoi ordini

.. con tutto il cuore

lo cercano,

$+{ }^{3}$ Mai

non hanno commesso l'iniquità,

.. nelle sue VIE

CAMMINANO.

(Sal 119,1-3)

I primi due segmenti iniziano con la stessa parola "Beati" (fungono da "ter nini iniziali"); "legge" e "ordini" sono sinonimi.

L'ultimo segmento dice la stessa cosa ma con la negazione: "l'iniquità" si o spone a "la legge" e a "gli ordini".

I segmenti estremi corrispondono, con la ripresa di "via/vie" e "camminare" (svolgono la funzione di "termini estremi" o "inclusione").

Soluzione del terzo esercizio:

$={ }^{6}{ }^{\circ} \overline{\mathrm{a}} \mathrm{z}$

$: b^{e} h a b b \hat{t} t \hat{\imath}$

$={ }^{7}$, ôdkā

$: b^{e}$ lomdî

$={ }^{8}$ 'et-huqqè̀āà

:: 'al-ta'azbēnî

$+{ }^{6}$ Allora

: guardando

+7 Ti loderò

: istruito

$=8$ I tuoi decreti

:: non abbandonarmi 1ō'-'ēbôš

'el-kol-

$\underline{\text { mișwōtèkā }}$

$b^{\mathrm{e} y o ̄ s ̌ e r ~}$

lēbāb

mišp têe șidqekā

'ešmōr

'ad-m ${ }^{\mathrm{e}} \overline{\mathrm{od}}$

non mi vergognerò

verso tutti

i tuoi comandamenti.

in rettitudine

dei giudizi

di cuore,

della tua giustizia.

osserverò,

completamente.

(Sal 119,6-8) 
I primi due segmenti si corrispondono: i loro primi membri sono complementari (lungi dal "vergognarmi", "loderò"), i loro secondi membri dicono il come, cioè l'osservanza della legge. Il terzo segmento è diverso: il suo primo membro corrisponde al secondo membro dei primi due segmenti (l'osservanza della legge), il suo secondo membro chiama l'aiuto di Dio, come per dire che ha bisogno dell'aiuto del Signore per osservare la sua legge. ${ }^{21}$

Soluzione del quarto esercizio:

\begin{tabular}{|c|c|c|}
\hline \multirow{3}{*}{$\begin{array}{l}+{ }^{1} \text { 'ašrêê } \\
: \text { hahōlkîm } \\
+{ }^{2} \text { 'ašrêê } \\
: b^{\mathrm{e}} \text { kol-lēb } \\
-^{3} \text { 'ap } \\
: \text { bidrākāyw }\end{array}$} & $\begin{array}{l}\text { temîmê- } \\
\text { b }^{\mathrm{e}} \underline{\text { tôrat }}\end{array}$ & $\begin{array}{l}\text { dārek } \\
\text { yhwh }\end{array}$ \\
\hline & $\begin{array}{l}\text { nōșrê } \\
\text { yidrěsûhûu }\end{array}$ & ‘ēdōtāyw \\
\hline & $\begin{array}{l}\text { lō'-pā'ălû } \\
\boldsymbol{h} \bar{a} l \bar{a} \boldsymbol{k} \hat{u}\end{array}$ & 'awlâ \\
\hline $\begin{array}{c}-{ }^{4} \text { ' attâ } \\
\quad:: \text { lišmōr }\end{array}$ & $\begin{array}{l}\text { șiwwîtâ } \\
\text { me' }^{\mathbf{e}} \overline{\mathbf{o d}}\end{array}$ & piqqudèkā \\
\hline $\begin{array}{l}\text { - }{ }^{5} \text {, ahăălay } \\
\quad:: \text { lišmōr }\end{array}$ & $\begin{array}{l}\text { yikkōnû } \\
\text { huqqèkāa }\end{array}$ & derākāy \\
\hline$={ }^{6}{ }^{\prime} \bar{a} z$ & lō'-'ēbôš & miswōtèkā \\
\hline $\begin{array}{l}={ }^{7} \text { 'ôdkā } \\
: b^{\mathrm{e}} \text { lomdî }\end{array}$ & $\begin{array}{l}b^{\mathrm{e} y o ̄ s ̌ e r} \\
\text { mišp tê }\end{array}$ & $\begin{array}{l}\text { lēbāb } \\
\text { șidqekā }\end{array}$ \\
\hline $\begin{array}{l}={ }^{8} \text { 'et-huqqèkāa } \\
: \text { 'al-ta'azbēnî }\end{array}$ & $\begin{array}{l}\text { 'ešmōr } \\
\text { 'ad-me, } \overline{\mathbf{o}} \mathbf{d}\end{array}$ & \\
\hline
\end{tabular}

${ }^{21}$ Vedi la mia analisi retorica del Sal 119 in MEYNET, R., Le Psautier, p. 189-325. 


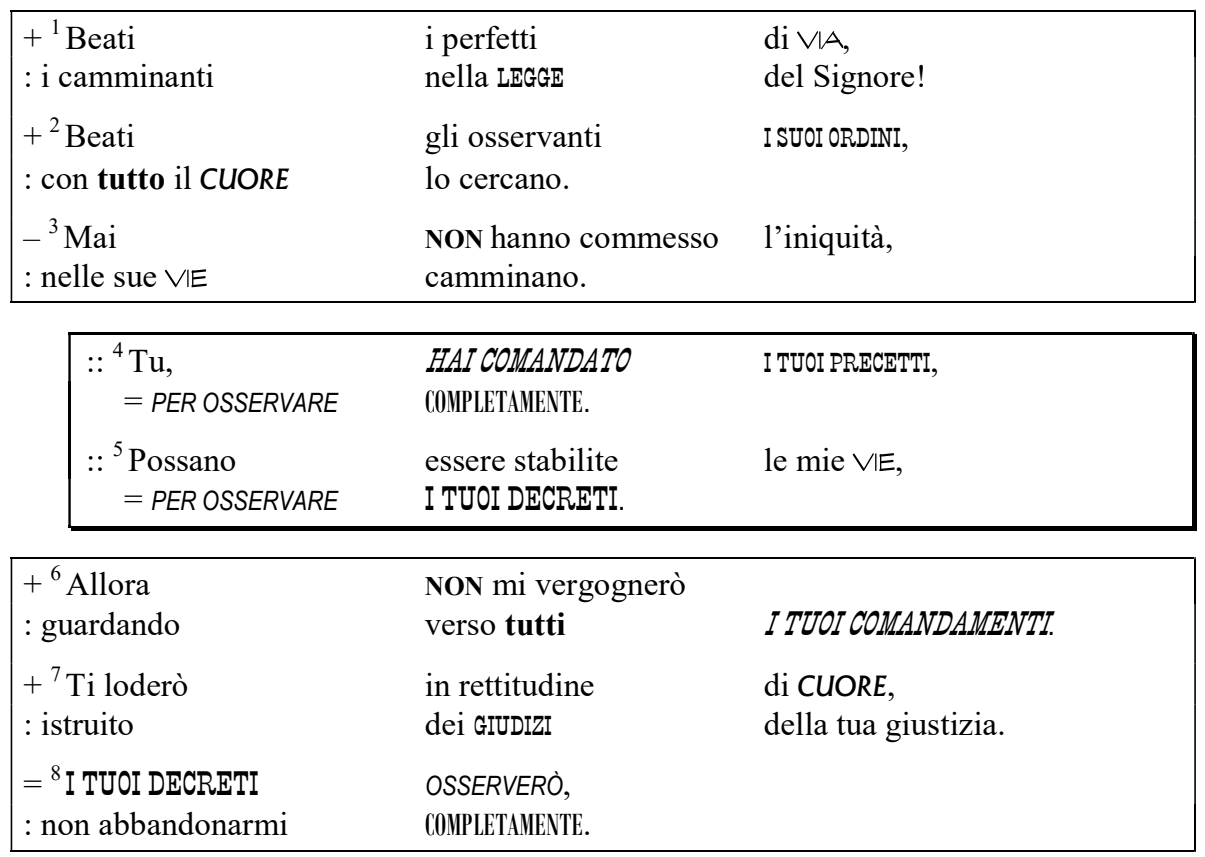

La parte centrale, come spesso accade, occupa un gran numero di termini che si trovano negli altri due. Ha in comune con l'ultima parte, "tu hai comandato" e "i tuoi comandamenti" (4a.6b), "osservare" (4b.5b.8a), "totalmente" (4b.8b), "i tuoi decreti "(5b.8a); "Vie" di 5a si trova già agli estremi della prima parte (1a.3b).

"Cuore" ritorna nei segmenti centrali delle parti estreme (2b.7a); "tutto" di $2 \mathrm{~b}$ sarà ripreso da "tutti" in $6 \mathrm{~b}$. Infine, notiamo che nell'ultimo segmento della prima parte e nel primo segmento dell'ultima, i secondi termini sono i verbi con la negazione (3a.6a).

La prima parte, che è tutta alla terza persona plurale, elogia i giusti che osservano la Legge di Dio (1-2) evitando ogni iniquità (3). La terza parte è in prima persona singolare, come se il salmista volesse contare se stesso tra i giusti della prima parte. Proprio come i giusti "non hanno commesso iniquità", lui "non si vergognerà". I due segmenti della parte centrale collegano il Signore che ha dato i suoi decreti da osservare (4) e il salmista che chiede di poterlo fare (5). ${ }^{22}$

${ }^{22}$ MEYNET, R., Le Psautier, p. 195-197. 
Soluzione del quinto esercizio:

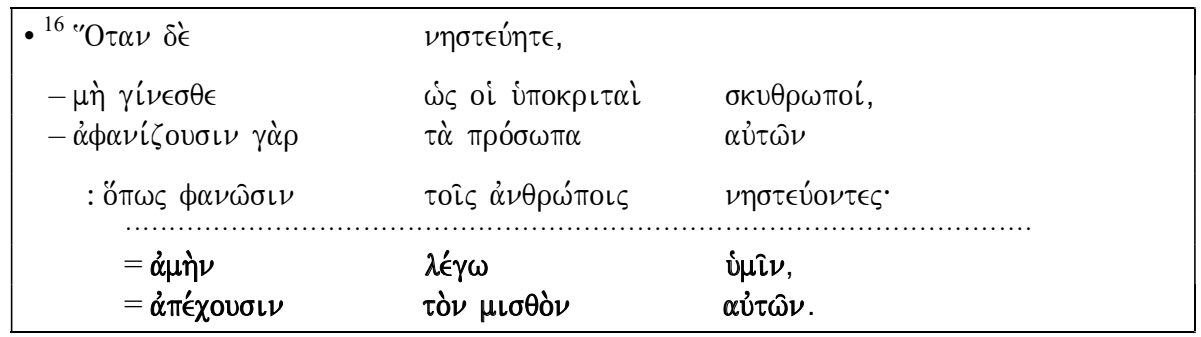

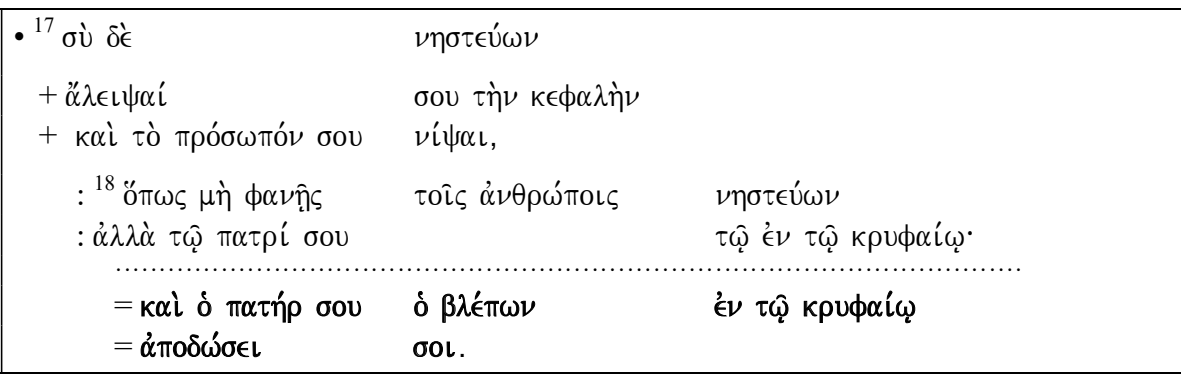

\begin{tabular}{|c|c|c|}
\hline - ${ }^{16}$ QUANDO & DIGIUNATE, & \\
\hline - non siate & come gli ipocriti & DAL VOLTO-SCURO: \\
\hline - scompongono infatti & LE FACCE & LORO, \\
\hline : affinché appaiano & agli uomini & DIGIUNANDO. \\
\hline $\begin{array}{l}=\text { In verità } \\
=\text { tengono }\end{array}$ & $\begin{array}{l}\text { dico } \\
\text { la ricompensa }\end{array}$ & $\begin{array}{l}\text { a voi, } \\
\text { di loro. }\end{array}$ \\
\hline
\end{tabular}

\begin{tabular}{|c|c|c|}
\hline - ${ }^{17}$ MA TU, & DIGIUNANDO, & \\
\hline+ profuma & LA TUA TESTA & \\
\hline + e LA TUA FACCIA & lava, & \\
\hline $\begin{array}{l}:{ }^{18} \text { affinché tu non appaia } \\
\text { : ma al tuo Padre }\end{array}$ & agli uomini & $\begin{array}{l}\text { DIGIUNANDO, } \\
\text { che (è) nel segreto. }\end{array}$ \\
\hline $\begin{array}{l}=\mathbf{E} \text { il tuo Padre } \\
=\text { ridarà in cambio }\end{array}$ & $\begin{array}{l}\text { che vede } \\
\text { a te. }\end{array}$ & nel segreto \\
\hline
\end{tabular}




\section{Riferimenti bibliografici}

BOYS, T. A Key to the Book of the Psalms. L.B Seeley \& Sons, London: Seeley, 1825.

BOYS, T. Tactica Sacra: An Attempt to Develope and to Exhibit to the Eye by Tabular Arrangements, a General Rule of Composition Prevailing in the Holy Scriptures. T. Hamilton, London: T. Hamilton, 1824. Disponibile in: <www.retoricabiblicaesemitica.org>. Visitato in: 13 ott. 2019.

FORBES, J. The Symmetrical of Scripture: or the principles of Scripture parallelism exemplified, in an analysis of the Decalogue, the Sermon on the Mount, and other passages of the sacred volume. Edinburgh: T\&T Clark, 1854. Disponibile in: <www.retoricabiblicaesemitica.org >. Visitato in: 13 ott. 2019.

JEBB, J. Sacred Literature: comprising a review of the principles of composition laid down by the late Robert Lowth, Lord Bishop of London in his Praelectiones and Isaiah: and an application of the principles so reviewed, to the illustration of the New Testament in a series of critical observations on the style and structure of that sacred volume. T.Cadell \& W. Davies, London: T. Cadell and W. Davies, 1820. Disponibile in: $<$ www.retoricabiblicaesemitica. org >. Visitato in: 13 ott. 2019.

KUGEL, J. K. The Idea of Biblical Poetry. Parallelism and Its History. Yale University Press, New Haven / London: Yale University, 1981.

LORI, G. Il discorso della montagna, dono del Padre (Mt 5,1-8,1). Bologna: EDB, 2013. (Retorica Biblica, 18).

LOWTH, R. De Sacra Poesi Hebraeorum: Praelectiones Academicae Oxonii Habitae. London: Claredon, 1753. Disponibile in: <www. retoricabiblicaesemitica.org>. Visitato in: 13 ott. 2019.

LUND, N. W. Chiasmus in the New Testament. A Study in Formgeschichte. The University of North Carolina Press, Chapel Hill: University of North Carolina Press, 1942.

LUND, N. W. Chiasmus in the New Testament: a Study in the Form and Function of Chiastic Structures. Hendrickson, Peabody (MA): Hendrickson Publishers, 1992.

MEYNET, R. A Análise retórica: um novo método para compreender a 
Bíblia. Brotéria, v.137, n.5, p. 391-408, 1993. Disponibile in: <www. retoricabiblicaesemitica.org>. Visitato in: 13 ott. 2019.

MEYNET, R. Histoire de «l'analyse rhétorique» en exégèse biblique. Rhetorica, v.8, n.4, p. 291-320, 1990. Disponibile in: <http://www. retoricabiblicaesemitica.org/arb_articolo_it.html>. Visitato in: 13 ott. 2019.

MEYNET, R. Historique. In: MEYNET, R. Traité de rhétorique biblique. Gabalda: Pendé, 2013. p. 31-110. (Rhétorique Sémitique, 11).

MEYNET, R. I frutti dell'analisi retorica. Gregorianum, v.77, n.3, p. 403436, 1996.

MEYNET, R. La composition du Notre Père. Studia Rhetorica Biblica et Semitica, n.18, p. 1-28, 2014. Disponibile in: <http://www. retoricabiblicaesemitica.org/Pubblicazioni/StRBS/18.NOTRE\%20PERE_ pagesLiturgie_14.12.16_Couleurs.pdf $>$. Visitato in: 13 ott. 2019.

MEYNET, R. La composizione del Padre nostro. Studia Rhetorica Biblica et Semitica, n.19, p. 1-20, 2015. Disponibile in: <http:// www.retoricabiblicaesemitica.org/Pubblicazioni/StRBS/19.La\%20 composizione\%20del\%20PN_12.07.2016.pdf>. Visitato in: 13 ott. 2019.

MEYNET, R. La rhétorique biblique et sémitique. Catholic Theology and Thought, n.72, p. 44-77, 2013.

MEYNET, R. La rhétorique biblique et sémitique. État de la question. Rhetorica, v.28, n.3, p. 290-312, 2010.

MEYNET, R. Le Psautier. Cinquième livre (Ps 107-150). Leuven: Peeters, 2017. (Rhetorica Biblica et Semitica, 12).

MEYNET, R. Première partie: les textes fondateurs. In: MEYNET, R. L'Analyse rhétorique. Une nouvelle méthode pour comprendre les textes bibliques: textes fondateurs et exposé systématique. Éd. du Cerf, Paris: Les Éditions du Cerf, 1989. p. 23-173. (Coll. Initiations).

MEYNET, R. Rhétorique biblique, rhétorique de l'énigme. Rhetorica, v.33, n.2, p. 147-180, 2015.

MEYNET, R. Traité de rhétorique biblique. Leuven: Peeters, 2013.

MEYNET, R. Une nouvelle présentation de la rhétorique biblique et sémitique. Exercices de rhétorique, n. 8, p. 1-11, 2017. Disponibile in: $<$ http://rhetorique. revues.org/486>. Visitato in: 13 ott. 2019. 
MORTARA GARAVELLI, B. Manuale di retorica. Milano / Italianistica: Studi Bompiani, 1991.

ONISZCZUK, J. L'analisi retorica biblica e semitica. Gregorianum, v.94, n.3, p. 479-501, 2013. Disponibile in: <https://www.academia.edu/17283355/ Lanalisi_retorica_biblica_e_semitica $>$. Visitato in: 13 ott. 2019.

Roland Meynet Docente de Teologia Bíblica na Faculdade de Teologia da Pontificia Università Gregoriana Roma - Itália

E-mail: r.meynet@unigre.it

Recebido em: 28/01/20

Aprovado em: 14/07/20 